\title{
Scenario A
}

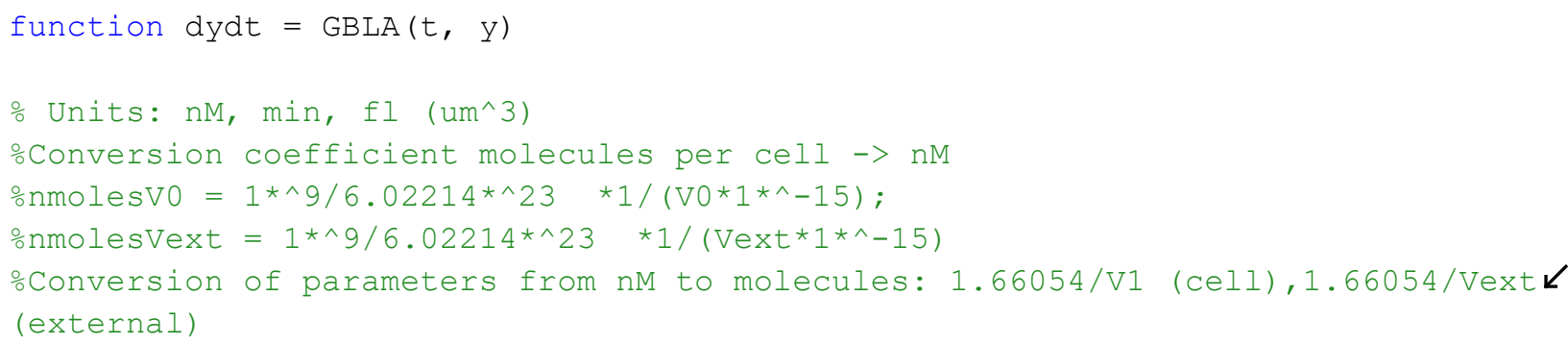

$\% \circ$

$\therefore$ chiset $=1$

$\mathrm{F}=\log (\mathrm{No})+\operatorname{umax} \cdot *(\mathrm{t}-\mathrm{l}+(\log (1-\exp (-\mathrm{v} \cdot \star \mathrm{t})+\exp (-\mathrm{v} \cdot *(\mathrm{t}-\mathrm{l}))) \cdot / \mathrm{v}))-(1 / \mathrm{m}) \cdot{ }^{*} \log (1+((\exp (\mathrm{m} \cdot \boldsymbol{k}$ $\left.\left.\left.\left.{ }^{\star} \operatorname{umax} .{ }^{*}\left(t-l+\left(\log \left(1-\exp \left(-\mathrm{v} \cdot{ }^{*} \mathrm{t}\right)+\exp \left(-\mathrm{v} \cdot{ }^{\star}(\mathrm{t}-\mathrm{l})\right)\right) \cdot / \mathrm{v}\right)\right)\right)-1\right) /\left(\exp \left(\mathrm{m} \cdot{ }^{*}(\log (\mathrm{K})-\log (\mathrm{No}))\right)\right)\right)\right)$; $\mathrm{N}=\exp (\mathrm{F}) ;$

$\mathrm{u}=\operatorname{umax} \cdot{ }^{*}\left(\left(\mathrm{v} \cdot{ }^{\star} \exp \left(-\mathrm{t} \cdot{ }^{\star} \mathrm{v}\right)-\mathrm{v} \cdot{ }^{*} \exp \left(\mathrm{v} \cdot{ }^{*}(1-\mathrm{t})\right)\right) \cdot /\left(\mathrm{v} \cdot{ }^{*}\left(\exp \left(\mathrm{v} \cdot{ }^{*}(1-\mathrm{t})\right)-\exp \left(-\mathrm{t} \cdot{ }^{*} \mathrm{v}\right)+1\right)\right)\right.$ $+1)-\left(\operatorname{umax} \cdot{ }^{*} \exp \left(\mathrm{m} \cdot{ }^{*} \operatorname{umax} \cdot{ }^{*}\left(t-1+\log \left(\exp \left(\mathrm{v} \cdot{ }^{*}(\boldsymbol{l}-\mathrm{t})\right)-\exp \left(-\mathrm{t} \cdot{ }^{*} \mathrm{v}\right)+1\right) \cdot / \mathrm{v}\right)\right){ }^{\star} \exp (-\mathrm{m} \cdot \boldsymbol{K}\right.$ $\left.{ }^{*}(\log (\mathrm{K})-\log (\mathrm{NO}))\right) \cdot{ }^{*}\left(\left(\mathrm{v} \cdot{ }^{*} \exp \left(-\mathrm{t} \cdot{ }^{*} \mathrm{~V}\right)-\mathrm{v} \cdot{ }^{*} \exp \left(\mathrm{v} \cdot{ }^{*}(1-\mathrm{t})\right)\right) \cdot /\left(\mathrm{v} \cdot{ }^{*}\left(\exp \left(\mathrm{v}^{*}(1-\mathrm{t})\right)-\exp \boldsymbol{L}\right.\right.\right.$ $\left.\left.\left.\left.\left(-\mathrm{t} \cdot{ }^{\star} \mathrm{V}\right)+1\right)\right)+1\right)\right) \cdot /\left(\exp \left(-\mathrm{m} \cdot{ }^{*}(\log (\mathrm{K})-\log (\mathrm{No}))\right) \cdot{ }^{*}\left(\exp \left(\mathrm{m} \cdot{ }^{*} \operatorname{umax} \cdot{ }^{*}\left(\mathrm{t}-1+\log \left(\exp \left(\mathrm{v} \cdot{ }^{\star} \boldsymbol{L}\right.\right.\right.\right.\right.\right.$ $\left.\left.\left.\left.(1-t))-\exp \left(-t \cdot{ }^{*} \mathrm{v}\right)+1\right) \cdot(\mathrm{v})\right)-1\right)+1\right) ;$

$\mathrm{Vo}=1.2$

$\mathrm{VC}$ tot $=\mathrm{N} * \mathrm{Vo}$

Vtot $=1.8 e+15$;

$\mathrm{Vext}=\mathrm{Vtot}-\mathrm{VC}_{\mathrm{C}}$ tot;

$\mathrm{Vx}=\mathrm{Vext} . / \mathrm{VC}_{\mathrm{C}}$ tot;

$r=1 . / V x$;

$\mathrm{kFR}=\mathrm{kF}{ }^{*} \mathrm{chi}$;

\% $\mathrm{kFR}=\mathrm{kF}^{*}$ chiset;

$\mathrm{kFA}=\mathrm{kF} / \mathrm{chi}$; 


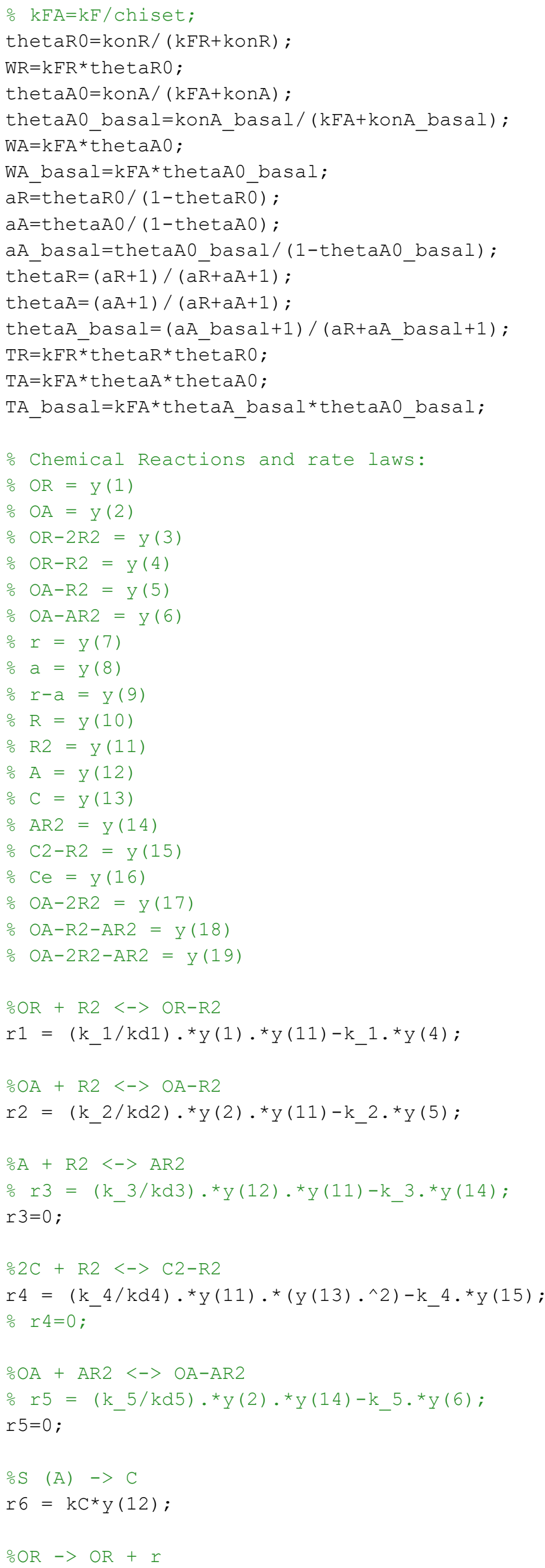




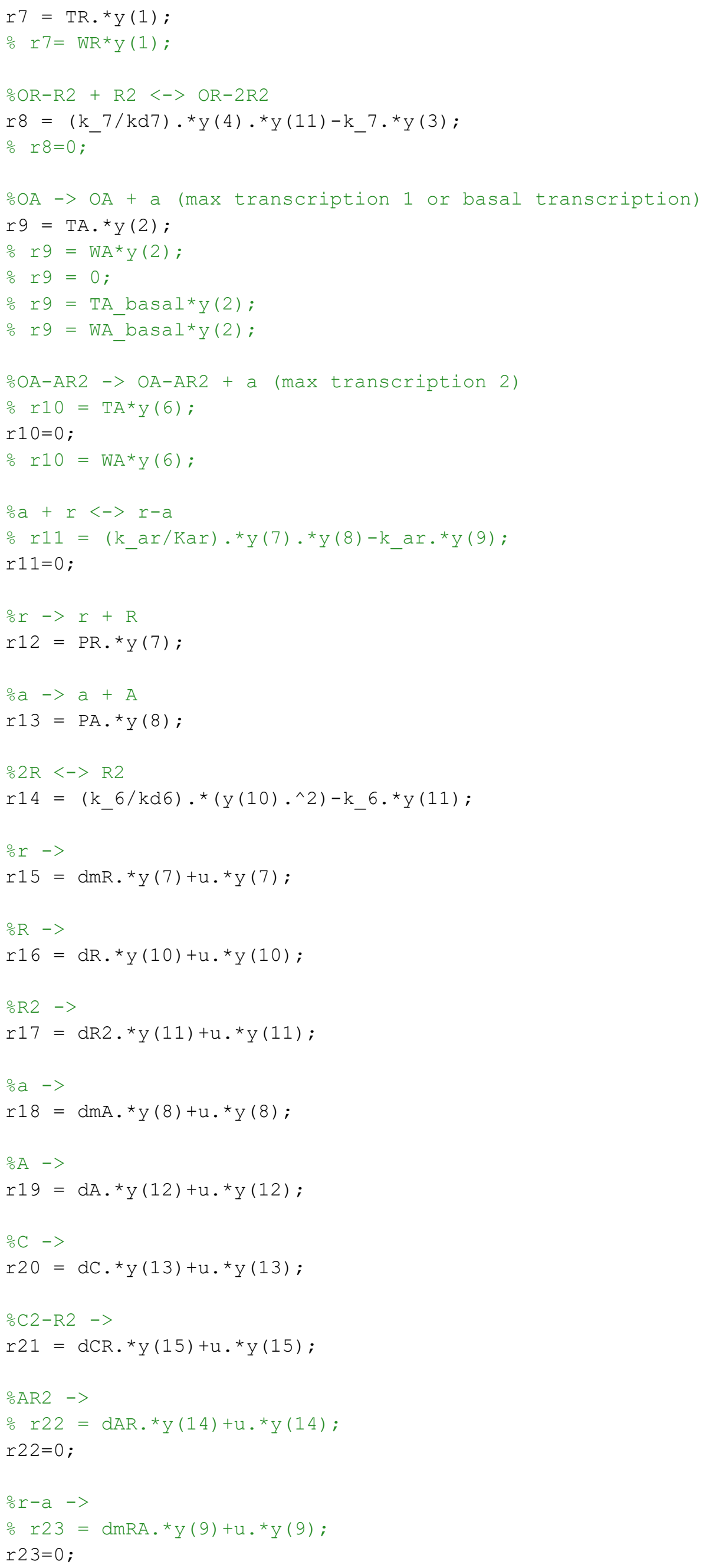




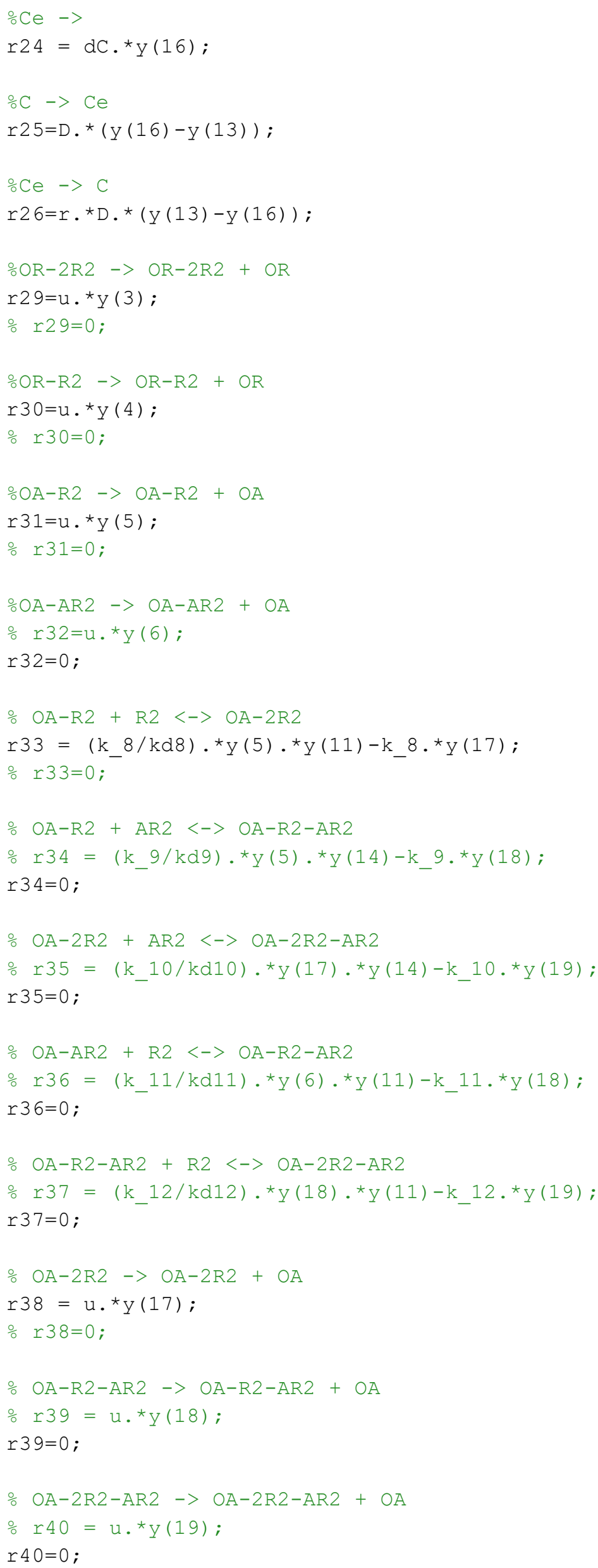


oDifferential equations:

$d y d t=[r 30-r 1+r 29 ; \ldots . \circ \mathrm{OR}$

$r 31+r 32+r 38+r 39+r 40-r 2-r 5 ; \ldots \circ O A$

$r 8-r 29 ; \ldots \% \mathrm{OR}-2 \mathrm{R} 2$

$r 1-r 8-r 30 ; \ldots \% \mathrm{OR}-\mathrm{R} 2$

$r 2-r 31-r 33-r 34 ; \ldots . \circ \mathrm{OA}-\mathrm{R} 2$

$r 5-r 32-r 36 ; \ldots \circ \mathrm{OA}-\mathrm{AR} 2$

$r 7-r 11-r 15 ; \ldots \circ \div$

$r 9+r 10-r 11-r 18 ; \ldots$.

$r 11-r 23 ; \ldots \circ \frac{\circ}{\circ}-a$

$r 12-r 14-r 16 ; \ldots \circ \mathrm{R}$

$r 14-r 1-r 2-r 3-r 4-r 8-r 17-r 33-r 36-r 37 ; \ldots$ ․ R2

$r 13-r 3-r 19 ; \ldots$. $\mathrm{A}$

$r 6-r 4-r 20+r 25 ; \ldots$. $\mathrm{C}$

$r 3-r 5-r 22-r 34-r 35 ; \ldots$ AR2

$r 4-r 21 ; \ldots \circ \mathrm{C} 2-\mathrm{R} 2$

$r 26-r 24+r 42 ; \ldots \quad \div \mathrm{Ce}$

$r 33-r 35-r 38 ; \ldots \circ \mathrm{OA}-2 \mathrm{R} 2$

$r 34+r 36-r 37-r 39 ; \ldots$ O OA-R2-AR2

$r 35+r 37-r 40] ; \% O A-2 R 2-A R 2$

end 


\section{Scenario B}

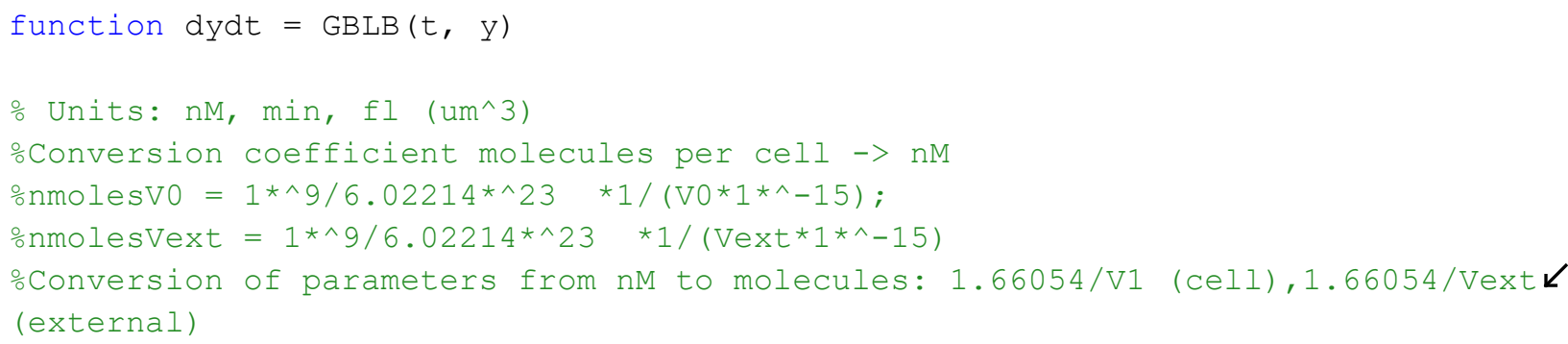

응 second set parameters values

global No $\mathrm{N} K \mathrm{l} \mathrm{V}$ m;

global u umax;

global r;

global Vo Vext Vtot VC tot Vx;

global TR TA TA_basal thetaR0 thetaR aR thetaA0 aA aA basal thetaA thetaA0_basal $\boldsymbol{}$ thetaA_basal kFA kFR;

$\% \frac{0}{\circ}$

chiset $=1$;

$\mathrm{F}=\log (\mathrm{No})+\operatorname{umax} \cdot{ }^{*}\left(\mathrm{t}-\mathrm{l}+\left(\log \left(1-\exp \left(-\mathrm{v} \cdot{ }^{*} \mathrm{t}\right)+\exp \left(-\mathrm{v} \cdot{ }^{\star}(\mathrm{t}-\mathrm{l})\right)\right) \cdot / \mathrm{v}\right)\right)-(1 / \mathrm{m}) \cdot{ }^{*} \log (1+((\exp (\mathrm{m} \cdot /$ $\left.\left.\left.\left.{ }^{\star} \operatorname{umax} .{ }^{*}\left(t-l+\left(\log \left(1-\exp \left(-\mathrm{v} \cdot{ }^{*} \mathrm{t}\right)+\exp \left(-\mathrm{v} \cdot{ }^{\star}(\mathrm{t}-\mathrm{l})\right)\right) \cdot / \mathrm{v}\right)\right)\right)-1\right) /\left(\exp \left(\mathrm{m} \cdot{ }^{*}(\log (\mathrm{K})-\log (\mathrm{No}))\right)\right)\right)\right)$; $\mathrm{N}=\exp (\mathrm{F}) ;$

$\mathrm{u}=\operatorname{umax} \cdot{ }^{*}\left(\left(\mathrm{v} \cdot{ }^{\star} \exp \left(-\mathrm{t} \cdot{ }^{\star} \mathrm{v}\right)-\mathrm{v} \cdot{ }^{*} \exp \left(\mathrm{v} \cdot{ }^{*}(1-\mathrm{t})\right)\right) \cdot /\left(\mathrm{v} \cdot{ }^{*}\left(\exp \left(\mathrm{v} \cdot{ }^{*}(1-\mathrm{t})\right)-\exp \left(-\mathrm{t} \cdot{ }^{*} \mathrm{v}\right)+1\right)\right)\right.$ $+1)-\left(\operatorname{umax} \cdot{ }^{*} \exp \left(\mathrm{m} \cdot{ }^{*} \operatorname{umax} \cdot{ }^{*}\left(t-1+\log \left(\exp \left(\mathrm{v} \cdot{ }^{*}(\boldsymbol{l}-\mathrm{t})\right)-\exp \left(-\mathrm{t} \cdot{ }^{*} \mathrm{v}\right)+1\right) \cdot / \mathrm{v}\right)\right){ }^{\star} \exp (-\mathrm{m} \cdot \boldsymbol{K}\right.$ $\left.{ }^{*}(\log (\mathrm{K})-\log (\mathrm{NO}))\right) \cdot{ }^{*}\left(\left(\mathrm{v} \cdot{ }^{*} \exp \left(-\mathrm{t} \cdot{ }^{*} \mathrm{~V}\right)-\mathrm{v} \cdot{ }^{*} \exp \left(\mathrm{v} \cdot{ }^{*}(1-\mathrm{t})\right)\right) \cdot /\left(\mathrm{v} \cdot{ }^{*}\left(\exp \left(\mathrm{v}^{*}(1-\mathrm{t})\right)-\exp \boldsymbol{L}\right.\right.\right.$ $\left.\left.\left.\left.\left(-\mathrm{t} \cdot{ }^{\star} \mathrm{V}\right)+1\right)\right)+1\right)\right) \cdot /\left(\exp \left(-\mathrm{m} \cdot{ }^{*}(\log (\mathrm{K})-\log (\mathrm{No}))\right) \cdot{ }^{*}\left(\exp \left(\mathrm{m} \cdot{ }^{*} \operatorname{umax} \cdot{ }^{*}\left(\mathrm{t}-1+\log \left(\exp \left(\mathrm{v} \cdot{ }^{\star} \boldsymbol{L}\right.\right.\right.\right.\right.\right.$ $\left.\left.\left.\left.(1-t))-\exp \left(-t \cdot{ }^{*} \mathrm{v}\right)+1\right) \cdot(\mathrm{v})\right)-1\right)+1\right) ;$

$\mathrm{Vo}=1.2 ;$

$\mathrm{VC}$ tot $=\mathrm{N} * \mathrm{Vo}$

Vtot $=1.8 e+15$;

$\mathrm{Vext}=\mathrm{Vtot}-\mathrm{VC}_{\mathrm{C}}$ tot;

$\mathrm{Vx}=\mathrm{Vext} . / \mathrm{V}_{\mathrm{C}}$ tot;

$r=1 . / V x$;

\% $\mathrm{kFR}=\mathrm{kF}^{*} \mathrm{chi}$;

$\mathrm{kFR}=\mathrm{kF}^{*}$ chiset ;

$\circ \mathrm{kFA}=\mathrm{kF} / \mathrm{chi}$; 


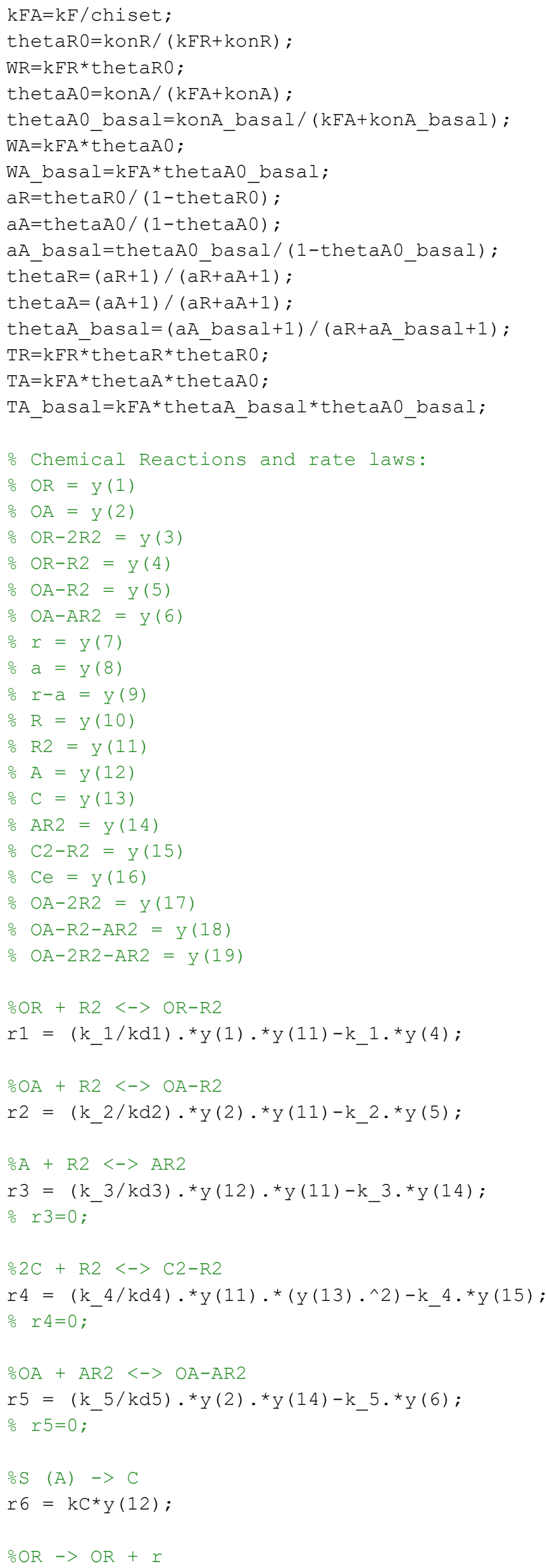




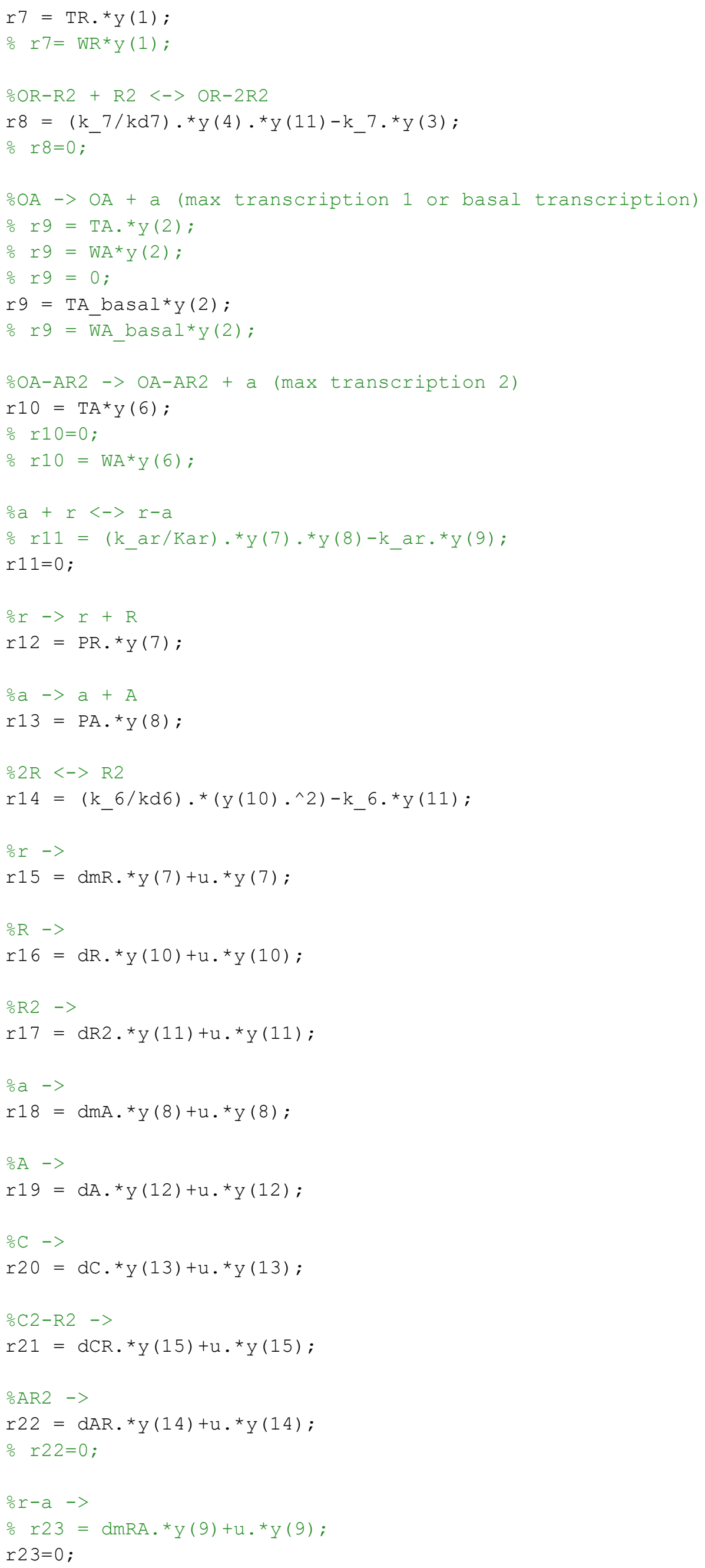




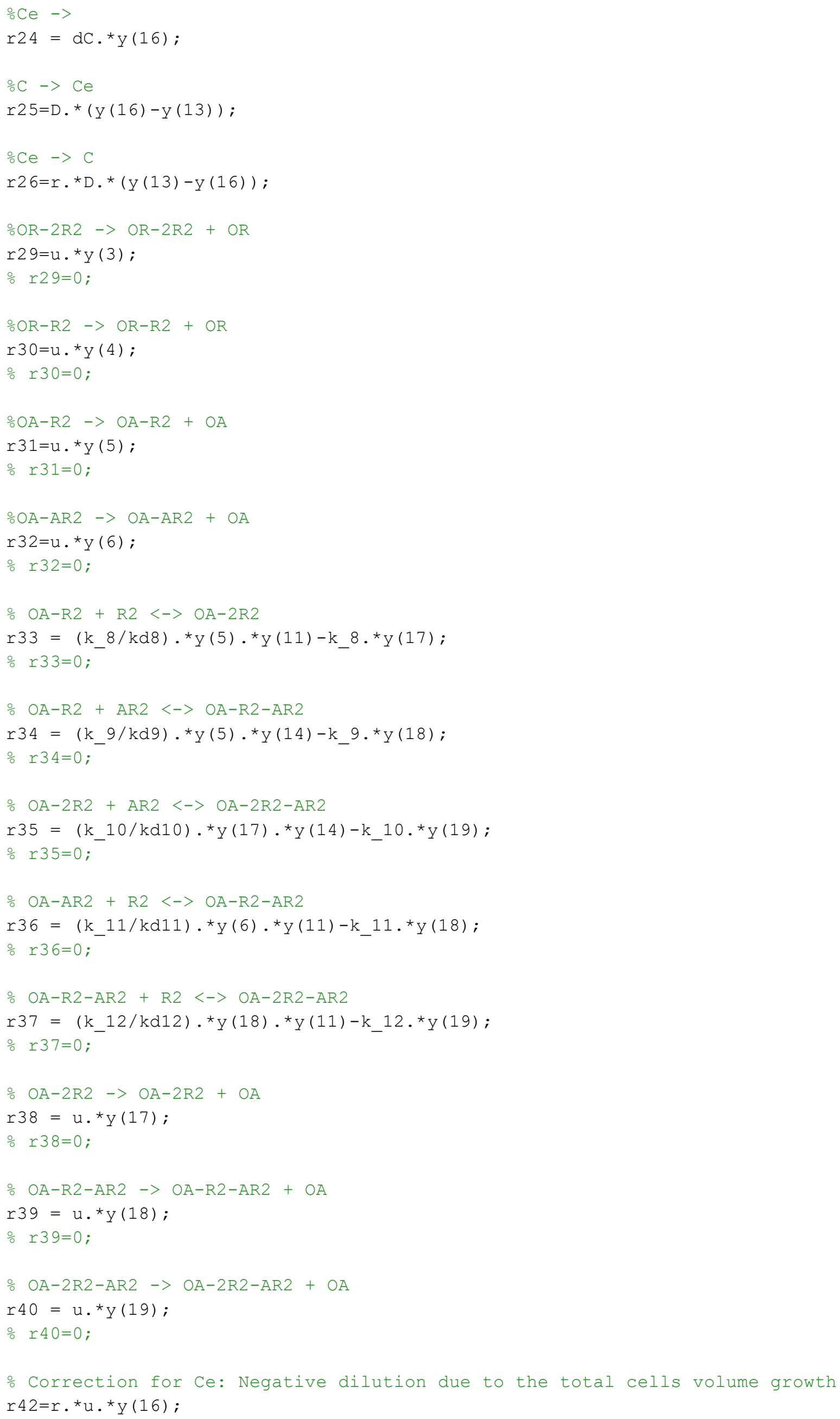


oDifferential equations:

$d y d t=[r 30-r 1+r 29 ; \ldots . \circ \mathrm{OR}$

$r 31+r 32+r 38+r 39+r 40-r 2-r 5 ; \ldots \circ O A$

$r 8-r 29 ; \ldots \% \mathrm{OR}-2 \mathrm{R} 2$

$r 1-r 8-r 30 ; \ldots \% \mathrm{OR}-\mathrm{R} 2$

$r 2-r 31-r 33-r 34 ; \ldots . \circ \mathrm{OA}-\mathrm{R} 2$

$r 5-r 32-r 36 ; \ldots \circ \mathrm{OA}-\mathrm{AR} 2$

$r 7-r 11-r 15 ; \ldots \circ \div$

$r 9+r 10-r 11-r 18 ; \ldots$.

$r 11-r 23 ; \ldots \circ \frac{\circ}{\circ}-a$

$r 12-r 14-r 16 ; \ldots \circ \mathrm{R}$

$r 14-r 1-r 2-r 3-r 4-r 8-r 17-r 33-r 36-r 37 ; \ldots$ ․ R2

$r 13-r 3-r 19 ; \ldots$. $\mathrm{A}$

$r 6-r 4-r 20+r 25 ; \ldots$. $\mathrm{C}$

$r 3-r 5-r 22-r 34-r 35 ; \ldots$ AR2

$r 4-r 21 ; \ldots \circ \mathrm{C} 2-\mathrm{R} 2$

$r 26-r 24+r 42 ; \ldots \quad \div \mathrm{Ce}$

$r 33-r 35-r 38 ; \ldots \circ \mathrm{OA}-2 \mathrm{R} 2$

$r 34+r 36-r 37-r 39 ; \ldots$ O OA-R2-AR2

$r 35+r 37-r 40] ; \% O A-2 R 2-A R 2$

end 


\section{Scenario C}

function dydt $=\operatorname{GBLC}(t, y)$

o Units: nM, min, fl (um^3)

oConversion coefficient molecules per cell -> nM

onmolesvo = 1*^9/6.02214*^23 *1/(V0*1*^-15);

onmolesVext $=1 * \wedge 9 / 6.02214 * \wedge 23 \quad * 1 /($ Vext* $1 * \wedge-15)$

oConversion of parameters from nM to molecules: $1.66054 / \mathrm{V} 1$ (cell), 1.66054/Vext $\boldsymbol{K}$ (external)

o\% first set Parameter values:

global kd1 kd2 kd3 kd4 kd5 kd6 kd7 kd8 kd9 kd10 kd11 kd12

global k_1 k_2 k_3 k_4 k_5 k_6 k_7 k_8 k_9 k_10 k_11 k_12

global konR WR;

global konA konA_basal WA WA_basal;

global kF chi chiset;

global Kar k_ar;

global dmR;

global dmA;

global PR;

global PA;

global dR dR2;

global dA;

global kC;

global dC;

global D;

global dCR;

global dAR;

global dmRA;

응 second set parameters values

global No N K l v m;

global u umax;

global r;

global Vo Vext Vtot Vc_tot Vx;

global TR TA TA_basal thetaR0 thetaR aR thetaA0 aA aA_basal thetaA thetaA0_basal $\boldsymbol{}$ thetaA_basal kFA kFR;

$\% \frac{0}{\circ}$

chiset $=1$;

$\mathrm{F}=\log (\mathrm{No})+\operatorname{umax} \cdot{ }^{*}\left(\mathrm{t}-\mathrm{l}+\left(\log \left(1-\exp \left(-\mathrm{v} \cdot{ }^{*} \mathrm{t}\right)+\exp \left(-\mathrm{v} \cdot{ }^{\star}(\mathrm{t}-\mathrm{l})\right)\right) \cdot / \mathrm{v}\right)\right)-(1 / \mathrm{m}) \cdot{ }^{*} \log (1+((\exp (\mathrm{m} \cdot /$

$\left.\left.\left.\left.\star \operatorname{umax} . *\left(t-1+\left(\log \left(1-\exp \left(-\mathrm{v} \cdot{ }^{*} \mathrm{t}\right)+\exp \left(-\mathrm{v} \cdot{ }^{*}(\mathrm{t}-\mathrm{l})\right)\right) \cdot / \mathrm{v}\right)\right)\right)-1\right) /(\exp (\mathrm{m} \cdot *(\log (\mathrm{K})-\log (\mathrm{No}))))\right)\right)$;

$\mathrm{N}=\exp (\mathrm{F}) ;$

$\mathrm{u}=\operatorname{umax} \cdot{ }^{*}\left(\left(\mathrm{v} \cdot{ }^{\star} \exp \left(-\mathrm{t} \cdot{ }^{\star} \mathrm{v}\right)-\mathrm{v} \cdot{ }^{*} \exp \left(\mathrm{v} \cdot{ }^{*}(1-\mathrm{t})\right)\right) \cdot /\left(\mathrm{v} \cdot{ }^{\star}\left(\exp \left(\mathrm{v} \cdot{ }^{*}(1-\mathrm{t})\right)-\exp \left(-\mathrm{t} \cdot{ }^{*} \mathrm{v}\right)+1\right)\right)\right.$ $+1)-\left(\operatorname{umax} \cdot{ }^{*} \exp \left(\mathrm{m} \cdot{ }^{*} \operatorname{umax} \cdot{ }^{*}\left(t-1+\log \left(\exp \left(\mathrm{v} \cdot{ }^{*}(1-\mathrm{t})\right)-\exp \left(-\mathrm{t} \cdot{ }^{*} \mathrm{v}\right)+1\right) \cdot / \mathrm{v}\right)\right){ }^{\star} \exp (-\mathrm{m} \cdot \boldsymbol{K}\right.$ $\left.{ }^{*}(\log (\mathrm{K})-\log (\mathrm{NO}))\right) \cdot{ }^{*}\left(\left(\mathrm{v} \cdot{ }^{*} \exp \left(-\mathrm{t} \cdot{ }^{*} \mathrm{~V}\right)-\mathrm{v} \cdot{ }^{*} \exp \left(\mathrm{v} \cdot{ }^{*}(1-\mathrm{t})\right)\right) \cdot /\left(\mathrm{v} \cdot{ }^{*}\left(\exp \left(\mathrm{v}^{*}(1-\mathrm{t})\right)-\exp \boldsymbol{L}\right.\right.\right.$ $\left.\left.\left.\left.\left(-\mathrm{t} \cdot{ }^{*} \mathrm{~V}\right)+1\right)\right)+1\right)\right) \cdot /\left(\exp \left(-\mathrm{m} \cdot{ }^{\star}(\log (\mathrm{K})-\log (\mathrm{No}))\right) \cdot{ }^{*}\left(\exp \left(\mathrm{m} \cdot{ }^{*} \operatorname{umax} \cdot{ }^{\star}\left(\mathrm{t}-1+\log \left(\exp \left(\mathrm{v} \cdot{ }^{\star} \boldsymbol{L}\right.\right.\right.\right.\right.\right.$ $\left.\left.\left.\left.(1-t))-\exp \left(-t \cdot{ }^{*} \mathrm{~V}\right)+1\right) \cdot(\mathrm{v})\right)-1\right)+1\right)$;

$\mathrm{Vo}=1.2 ;$

$\mathrm{VC}$ tot $=\mathrm{N}^{*} \mathrm{Vo}$

Vtot $=1.8 e+15$;

Vext $=$ Vtot $-V_{C}$ tot;

$\mathrm{Vx}=\mathrm{Vext} . / \mathrm{V}_{\mathrm{C}}$ tot;

$r=1 . / V x$;

\% $\mathrm{kFR}=\mathrm{kF}^{*} \mathrm{chi}$;

$\mathrm{kFR}=\mathrm{kF}{ }^{*}$ chiset;

ㅇ $\mathrm{kFA}=\mathrm{kF} / \mathrm{chi}$; 


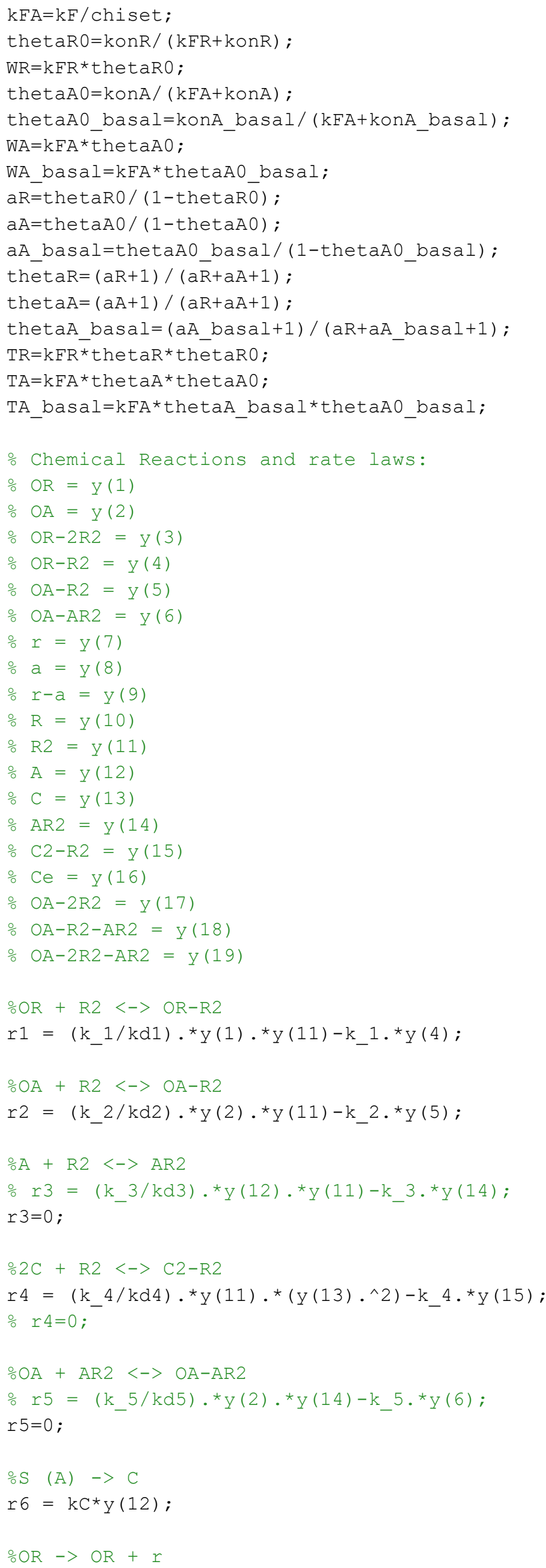




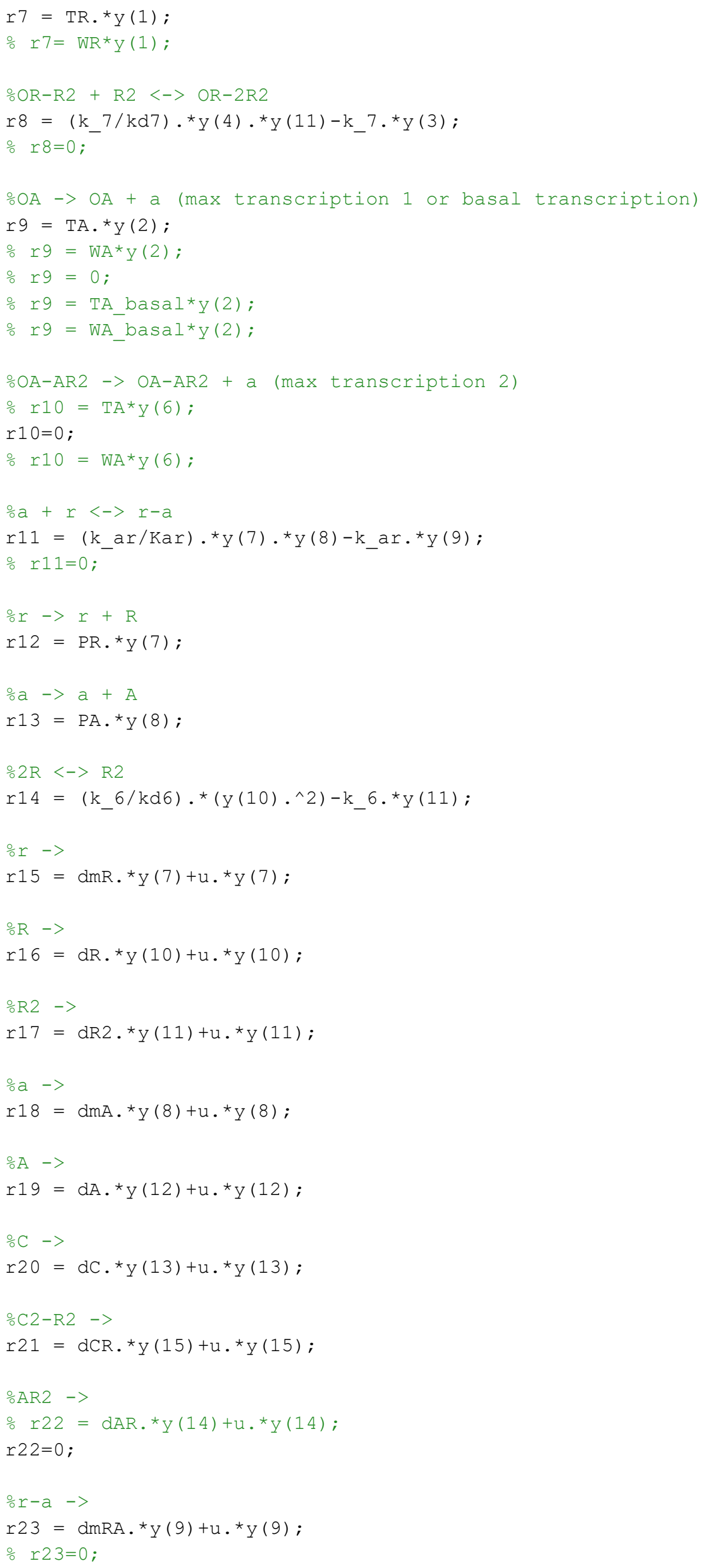




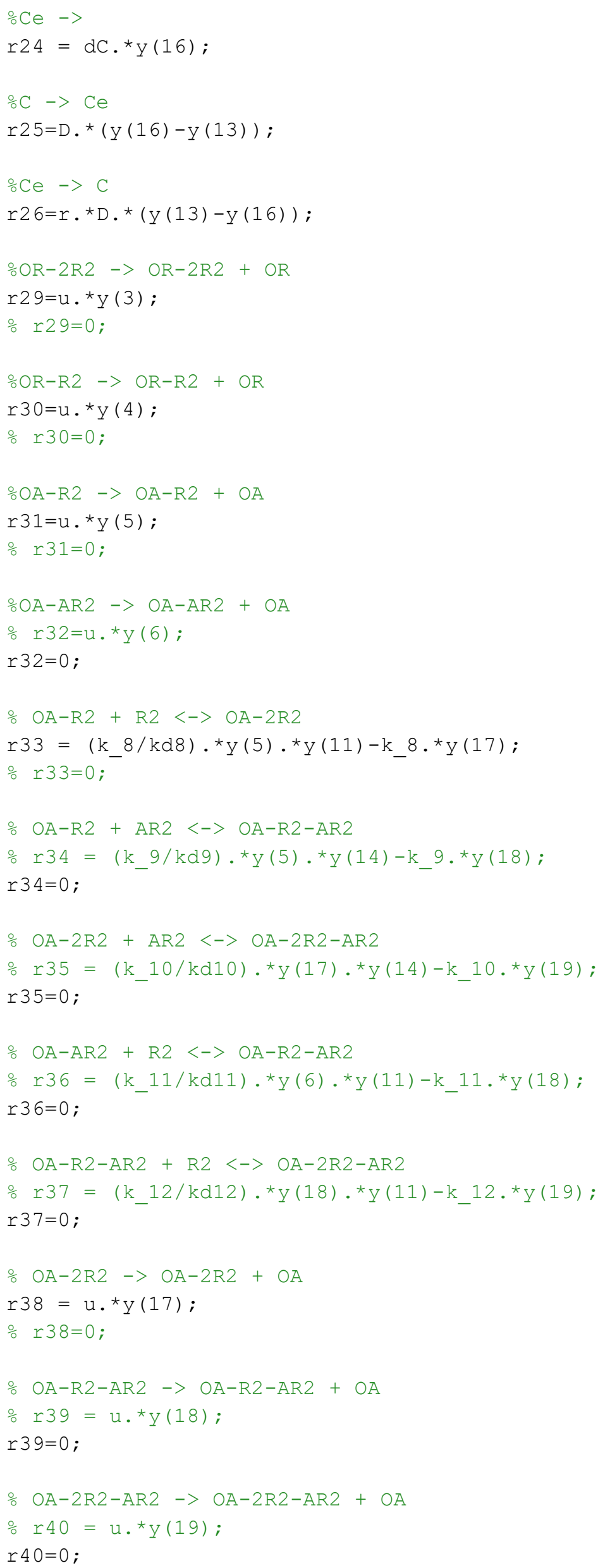


oDifferential equations:

$d y d t=[r 30-r 1+r 29 ; \ldots . \circ \mathrm{OR}$

$r 31+r 32+r 38+r 39+r 40-r 2-r 5 ; \ldots \circ O A$

$r 8-r 29 ; \ldots \% \mathrm{OR}-2 \mathrm{R} 2$

$r 1-r 8-r 30 ; \ldots \% \mathrm{OR}-\mathrm{R} 2$

$r 2-r 31-r 33-r 34 ; \ldots . \circ \mathrm{OA}-\mathrm{R} 2$

$r 5-r 32-r 36 ; \ldots \circ \mathrm{OA}-\mathrm{AR} 2$

$r 7-r 11-r 15 ; \ldots \circ \div$

$r 9+r 10-r 11-r 18 ; \ldots$.

$r 11-r 23 ; \ldots \circ \frac{\circ}{\circ}-a$

$r 12-r 14-r 16 ; \ldots \circ \mathrm{R}$

$r 14-r 1-r 2-r 3-r 4-r 8-r 17-r 33-r 36-r 37 ; \ldots$ ․ R2

$r 13-r 3-r 19 ; \ldots$. $\mathrm{A}$

$r 6-r 4-r 20+r 25 ; \ldots$. $\mathrm{C}$

$r 3-r 5-r 22-r 34-r 35 ; \ldots$ AR2

$r 4-r 21 ; \ldots \circ \mathrm{C} 2-\mathrm{R} 2$

$r 26-r 24+r 42 ; \ldots \quad \div \mathrm{Ce}$

$r 33-r 35-r 38 ; \ldots \circ \mathrm{OA}-2 \mathrm{R} 2$

$r 34+r 36-r 37-r 39 ; \ldots$ O OA-R2-AR2

$r 35+r 37-r 40] ; \% O A-2 R 2-A R 2$

end 


\section{Scenario D}

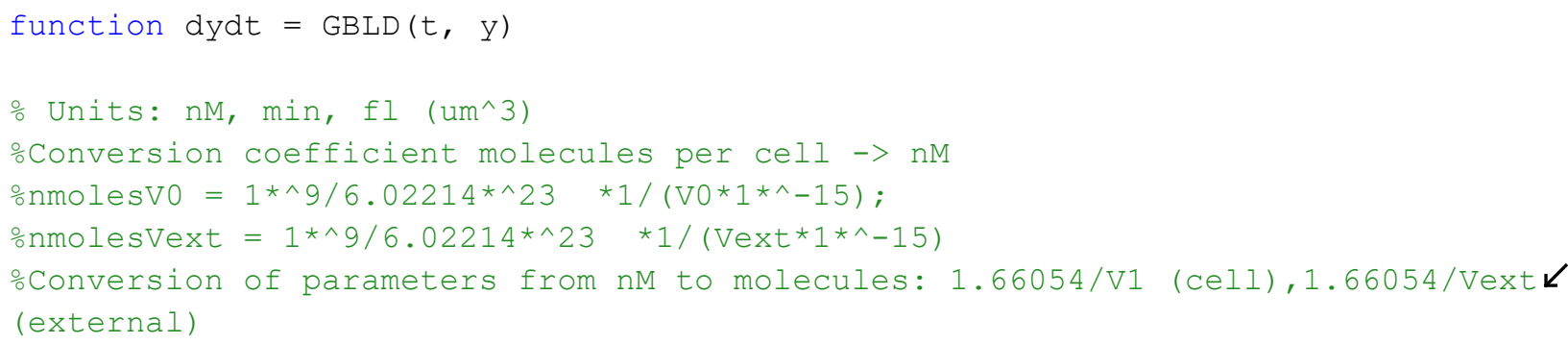

$\% \frac{0}{\circ}$

chiset $=1$;

$\mathrm{F}=\log (\mathrm{No})+\operatorname{umax} \cdot{ }^{*}\left(\mathrm{t}-\mathrm{l}+\left(\log \left(1-\exp \left(-\mathrm{v} \cdot{ }^{*} \mathrm{t}\right)+\exp \left(-\mathrm{v} \cdot{ }^{\star}(\mathrm{t}-\mathrm{l})\right)\right) \cdot / \mathrm{v}\right)\right)-(1 / \mathrm{m}) \cdot{ }^{*} \log (1+((\exp (\mathrm{m} \cdot /$ $\left.\left.\left.\left.\star \operatorname{umax} . *\left(t-1+\left(\log \left(1-\exp \left(-\mathrm{v} \cdot{ }^{*} \mathrm{t}\right)+\exp \left(-\mathrm{v} \cdot{ }^{*}(\mathrm{t}-\mathrm{l})\right)\right) \cdot / \mathrm{v}\right)\right)\right)-1\right) /(\exp (\mathrm{m} \cdot *(\log (\mathrm{K})-\log (\mathrm{No}))))\right)\right)$; $\mathrm{N}=\exp (\mathrm{F}) ;$

$\mathrm{u}=\operatorname{umax} \cdot{ }^{\star}\left(\left(\mathrm{v} \cdot{ }^{\star} \exp \left(-\mathrm{t} \cdot{ }^{*} \mathrm{v}\right)-\mathrm{v} \cdot{ }^{*} \exp \left(\mathrm{v} \cdot{ }^{*}(1-\mathrm{t})\right)\right) \cdot /\left(\mathrm{v} \cdot{ }^{*}\left(\exp \left(\mathrm{v} \cdot{ }^{*}(1-\mathrm{t})\right)-\exp \left(-\mathrm{t} \cdot{ }^{*} \mathrm{v}\right)+1\right)\right)\right.$ $+1)-\left(\operatorname{umax} \cdot{ }^{*} \exp \left(\mathrm{m} \cdot{ }^{*} \operatorname{umax} \cdot{ }^{*}\left(t-1+\log \left(\exp \left(\mathrm{v} \cdot{ }^{*}(1-\mathrm{t})\right)-\exp \left(-\mathrm{t} \cdot{ }^{*} \mathrm{v}\right)+1\right) \cdot / \mathrm{v}\right)\right){ }^{\star} \exp (-\mathrm{m} \cdot \boldsymbol{K}\right.$ $\left.{ }^{*}(\log (\mathrm{K})-\log (\mathrm{NO}))\right) \cdot{ }^{*}\left(\left(\mathrm{v} \cdot{ }^{*} \exp \left(-\mathrm{t} \cdot{ }^{*} \mathrm{~V}\right)-\mathrm{v} \cdot{ }^{*} \exp \left(\mathrm{v} \cdot{ }^{*}(1-\mathrm{t})\right)\right) \cdot /\left(\mathrm{v} \cdot{ }^{*}\left(\exp \left(\mathrm{v}^{*}(1-\mathrm{t})\right)-\exp \boldsymbol{L}\right.\right.\right.$ $\left.\left.\left.\left.\left(-\mathrm{t} \cdot{ }^{\star} \mathrm{V}\right)+1\right)\right)+1\right)\right) \cdot /\left(\exp \left(-\mathrm{m} \cdot{ }^{*}(\log (\mathrm{K})-\log (\mathrm{No}))\right) \cdot{ }^{*}\left(\exp \left(\mathrm{m} \cdot{ }^{*} \operatorname{umax} \cdot{ }^{*}\left(\mathrm{t}-1+\log \left(\exp \left(\mathrm{v} \cdot{ }^{\star} \boldsymbol{L}\right.\right.\right.\right.\right.\right.$ $\left.\left.\left.\left.(1-t))-\exp \left(-t \cdot{ }^{*} \mathrm{v}\right)+1\right) \cdot(\mathrm{v})\right)-1\right)+1\right) ;$

$\mathrm{Vo}=1.2 ;$

$\mathrm{VC}$ tot $=\mathrm{N} * \mathrm{Vo}$

Vtot $=1.8 e+15$;

$\mathrm{Vext}=\mathrm{Vtot}-\mathrm{VC}_{\mathrm{C}}$ tot;

$\mathrm{Vx}=\mathrm{Vext} . / \mathrm{V}_{\mathrm{C}}$ tot;

$r=1 . / V x$;

\% $\mathrm{kFR}=\mathrm{kF}^{*} \mathrm{chi}$;

$\mathrm{kFR}=\mathrm{kF}^{*}$ chiset;

$\circ \mathrm{kFA}=\mathrm{kF} / \mathrm{chi}$; 


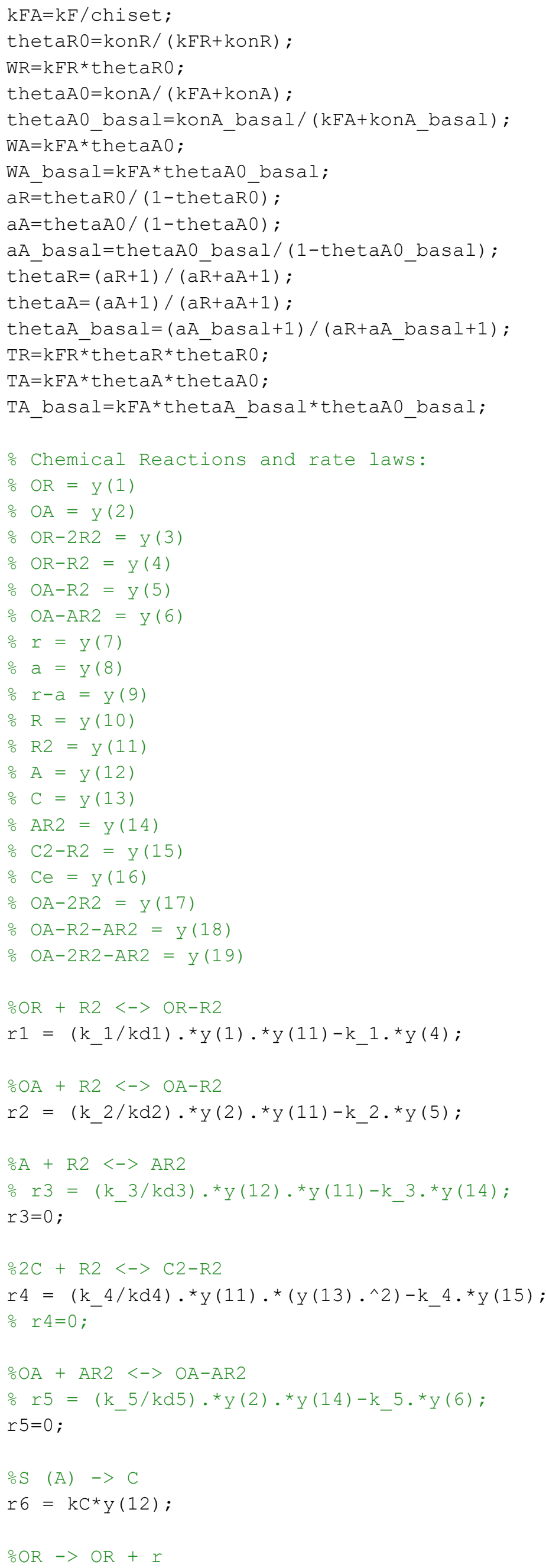




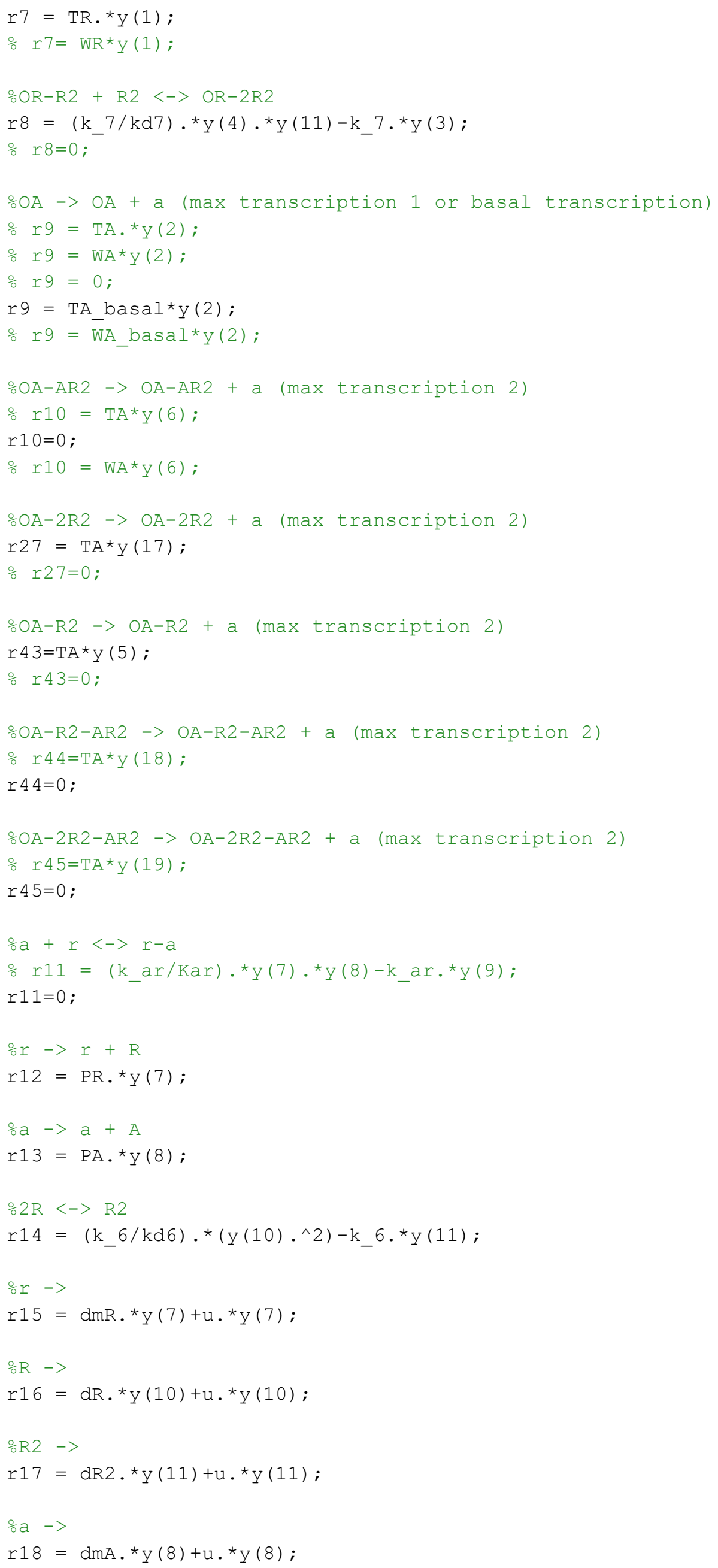




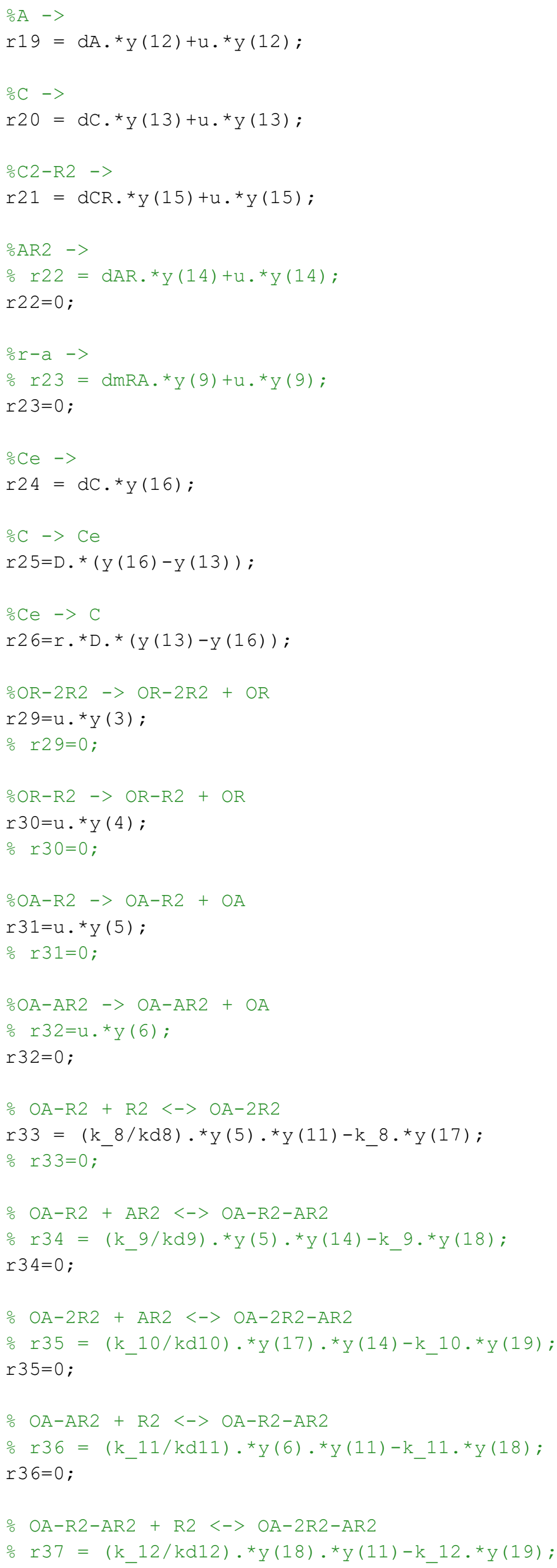


$r 37=0$

$\div \mathrm{OA}-2 \mathrm{R} 2->\mathrm{OA}-2 \mathrm{R} 2+\mathrm{OA}$

$\mathrm{r38}=\mathrm{u} \cdot{ }^{\star} \mathrm{y}(17)$;

당 0 ;

$\div \mathrm{OA}-\mathrm{R} 2-\mathrm{AR} 2->\mathrm{OA}-\mathrm{R} 2-\mathrm{AR} 2+\mathrm{OA}$

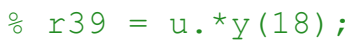

$r 39=0$

$\circ \mathrm{OA}-2 \mathrm{R} 2-\mathrm{AR} 2->\mathrm{OA}-2 \mathrm{R} 2-\mathrm{AR} 2+\mathrm{OA}$

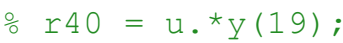

$r 40=0$;

o Correction for Ce: Negative dilution due to the total cells volume growth $r 42=r \cdot{ }^{*} u \cdot{ }^{\star} \mathrm{y}(16) ;$

Differential equations:

$d y d t=[r 30-r 1+r 29 ; \ldots . \circ \mathrm{OR}$

$r 31+r 32+r 38+r 39+r 40-r 2-r 5 ; \ldots \% O A$

$r 8-r 29 ; \ldots \circ \mathrm{OR}-2 \mathrm{R} 2$

$r 1-r 8-r 30 ; \ldots \circ \mathrm{OR}-\mathrm{R} 2$

$r 2-r 31-r 33-r 34 ; \ldots . \div \mathrm{OA}-\mathrm{R} 2$

$r 5-r 32-r 36 ; \ldots \circ \mathrm{OA}-\mathrm{AR} 2$

$r 7-r 11-r 15 ; \ldots \circ \mathrm{\circ}$

$r 9+r 10-r 11-r 18+r 27+r 43+r 44+r 45 ; \ldots$ a

$r 11-r 23 ; \ldots . . \div-a$

$r 12-r 14-r 16 ; \ldots \% \mathrm{R}$

$r 14-r 1-r 2-r 3-r 4-r 8-r 17-r 33-r 36-r 37 ; \ldots$ 응 2

$r 13-r 3-r 19 ; \ldots \circ \mathrm{A}$

$r 6-r 4-r 20+r 25 ; \ldots \circ C$

$r 3-r 5-r 22-r 34-r 35 ; \ldots \circ$ AR2

$r 4-r 21 ; \ldots \circ \mathrm{C} 2-\mathrm{R} 2$

$r 26-r 24+r 42 ; \ldots \quad \% \mathrm{Ce}$

$r 33-r 35-r 38 ; \ldots \circ \mathrm{OA}-2 \mathrm{R} 2$

$r 34+r 36-r 37-r 39 ; \ldots \circ \mathrm{OA}-\mathrm{R} 2-\mathrm{AR} 2$

$r 35+r 37-r 40] ; \div O A-2 R 2-A R 2$

end 


\section{Scenario $\mathrm{E}$}

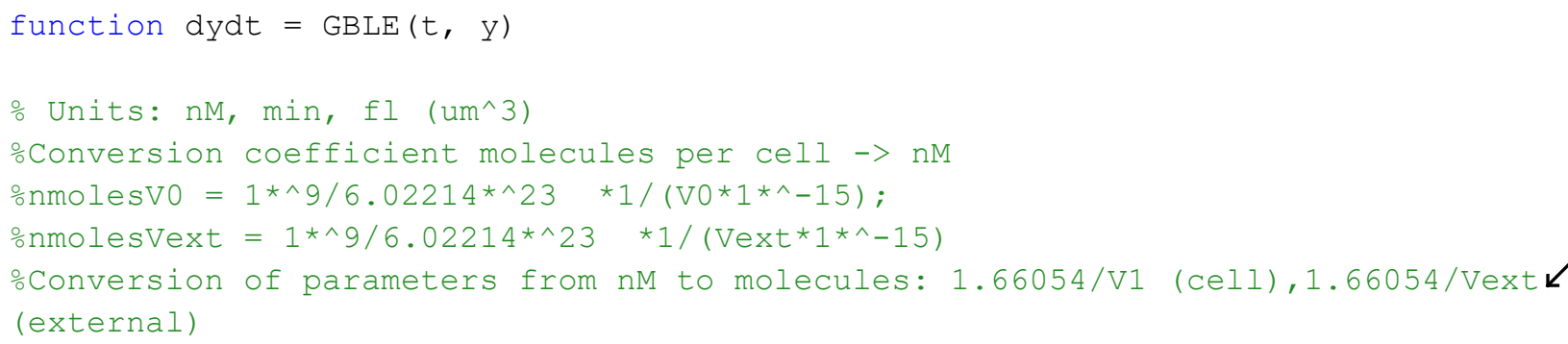

$\% \circ$

$\therefore$ chiset $=1$

$\mathrm{F}=\log (\mathrm{No})+\operatorname{umax} \cdot *(\mathrm{t}-\mathrm{l}+(\log (1-\exp (-\mathrm{v} \cdot \star \mathrm{t})+\exp (-\mathrm{v} \cdot *(\mathrm{t}-\mathrm{l}))) \cdot / \mathrm{v}))-(1 / \mathrm{m}) \cdot{ }^{*} \log (1+((\exp (\mathrm{m} \cdot \boldsymbol{k}$ $\left.\left.\left.\left.{ }^{\star} \operatorname{umax} .{ }^{*}\left(t-l+\left(\log \left(1-\exp \left(-\mathrm{v} \cdot{ }^{*} \mathrm{t}\right)+\exp \left(-\mathrm{v} \cdot{ }^{\star}(\mathrm{t}-\mathrm{l})\right)\right) \cdot / \mathrm{v}\right)\right)\right)-1\right) /\left(\exp \left(\mathrm{m} \cdot{ }^{*}(\log (\mathrm{K})-\log (\mathrm{No}))\right)\right)\right)\right)$; $\mathrm{N}=\exp (\mathrm{F}) ;$

$\mathrm{u}=\operatorname{umax} \cdot{ }^{*}\left(\left(\mathrm{v} \cdot{ }^{\star} \exp \left(-\mathrm{t} \cdot{ }^{\star} \mathrm{v}\right)-\mathrm{v} \cdot{ }^{*} \exp \left(\mathrm{v} \cdot{ }^{*}(1-\mathrm{t})\right)\right) \cdot /\left(\mathrm{v} \cdot{ }^{\star}\left(\exp \left(\mathrm{v} \cdot{ }^{*}(1-\mathrm{t})\right)-\exp \left(-\mathrm{t} \cdot{ }^{*} \mathrm{v}\right)+1\right)\right)\right.$ $+1)-\left(\operatorname{umax} \cdot{ }^{*} \exp \left(\mathrm{m} \cdot{ }^{*} \operatorname{umax} \cdot{ }^{*}\left(t-1+\log \left(\exp \left(\mathrm{v} \cdot{ }^{*}(\boldsymbol{l}-\mathrm{t})\right)-\exp \left(-\mathrm{t} \cdot{ }^{*} \mathrm{v}\right)+1\right) \cdot / \mathrm{v}\right)\right){ }^{\star} \exp (-\mathrm{m} \cdot \boldsymbol{K}\right.$ $\left.{ }^{*}(\log (\mathrm{K})-\log (\mathrm{NO}))\right) \cdot{ }^{*}\left(\left(\mathrm{v} \cdot{ }^{*} \exp \left(-\mathrm{t} \cdot{ }^{*} \mathrm{~V}\right)-\mathrm{v} \cdot{ }^{*} \exp \left(\mathrm{v} \cdot{ }^{*}(1-\mathrm{t})\right)\right) \cdot /\left(\mathrm{v} \cdot{ }^{*}\left(\exp \left(\mathrm{v}^{*}(1-\mathrm{t})\right)-\exp \boldsymbol{L}\right.\right.\right.$ $\left.\left.\left.\left.\left(-\mathrm{t} \cdot{ }^{\star} \mathrm{V}\right)+1\right)\right)+1\right)\right) \cdot /\left(\exp \left(-\mathrm{m} \cdot{ }^{*}(\log (\mathrm{K})-\log (\mathrm{No}))\right) \cdot{ }^{*}\left(\exp \left(\mathrm{m} \cdot{ }^{*} \operatorname{umax} \cdot{ }^{*}\left(\mathrm{t}-1+\log \left(\exp \left(\mathrm{v} \cdot{ }^{\star} \boldsymbol{L}\right.\right.\right.\right.\right.\right.$ $\left.\left.\left.\left.(1-t))-\exp \left(-t \cdot{ }^{*} \mathrm{v}\right)+1\right) \cdot(\mathrm{v})\right)-1\right)+1\right) ;$

$\mathrm{Vo}=1.2$

$\mathrm{VC}$ tot $=\mathrm{N} * \mathrm{Vo}$

Vtot $=1.8 e+15$;

Vext $=$ Vtot $-V_{C}$ tot;

$\mathrm{Vx}=\mathrm{Vext} . / \mathrm{VC}_{\mathrm{C}}$ tot;

$\mathrm{r}=1 . / \mathrm{Vx}$;

$\mathrm{kFR}=\mathrm{kF}{ }^{*} \mathrm{chi}$;

ㅇ $\mathrm{kFR}=\mathrm{kF}^{*}$ chiset;

$\mathrm{kFA}=\mathrm{kF} / \mathrm{chi}$; 


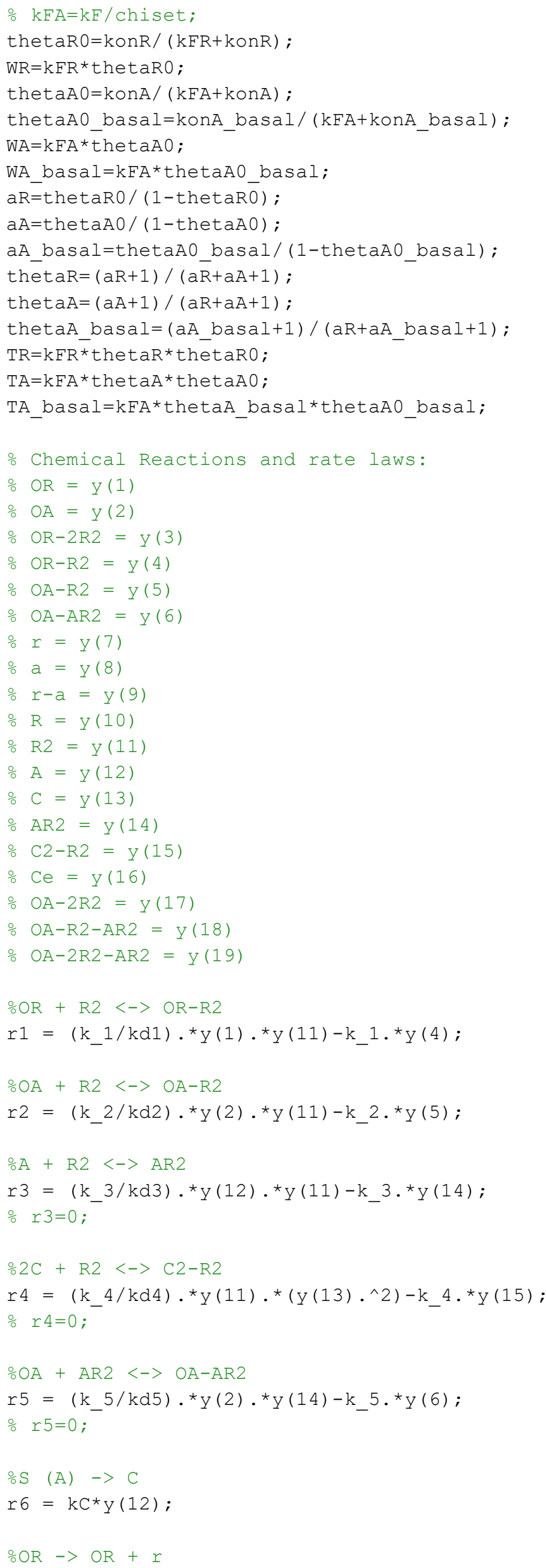




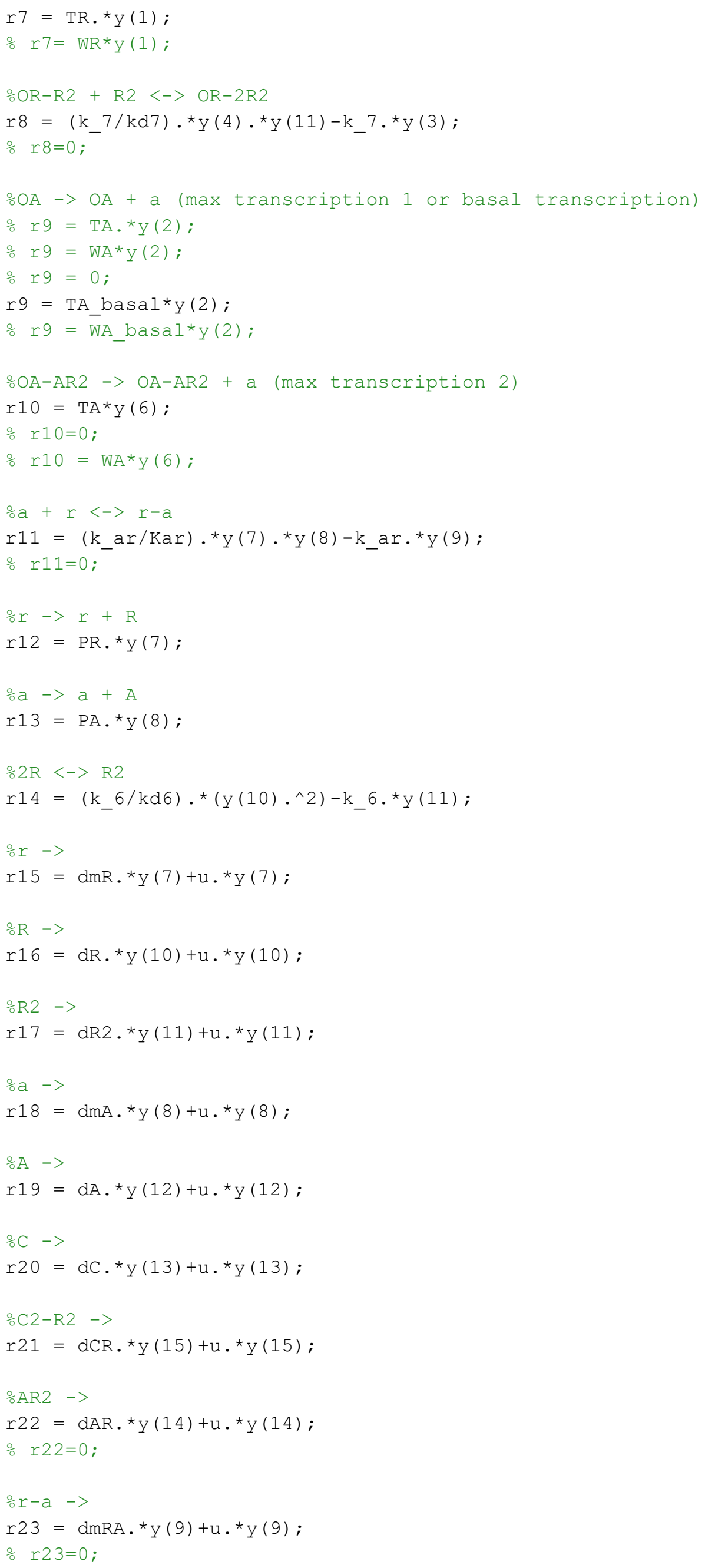




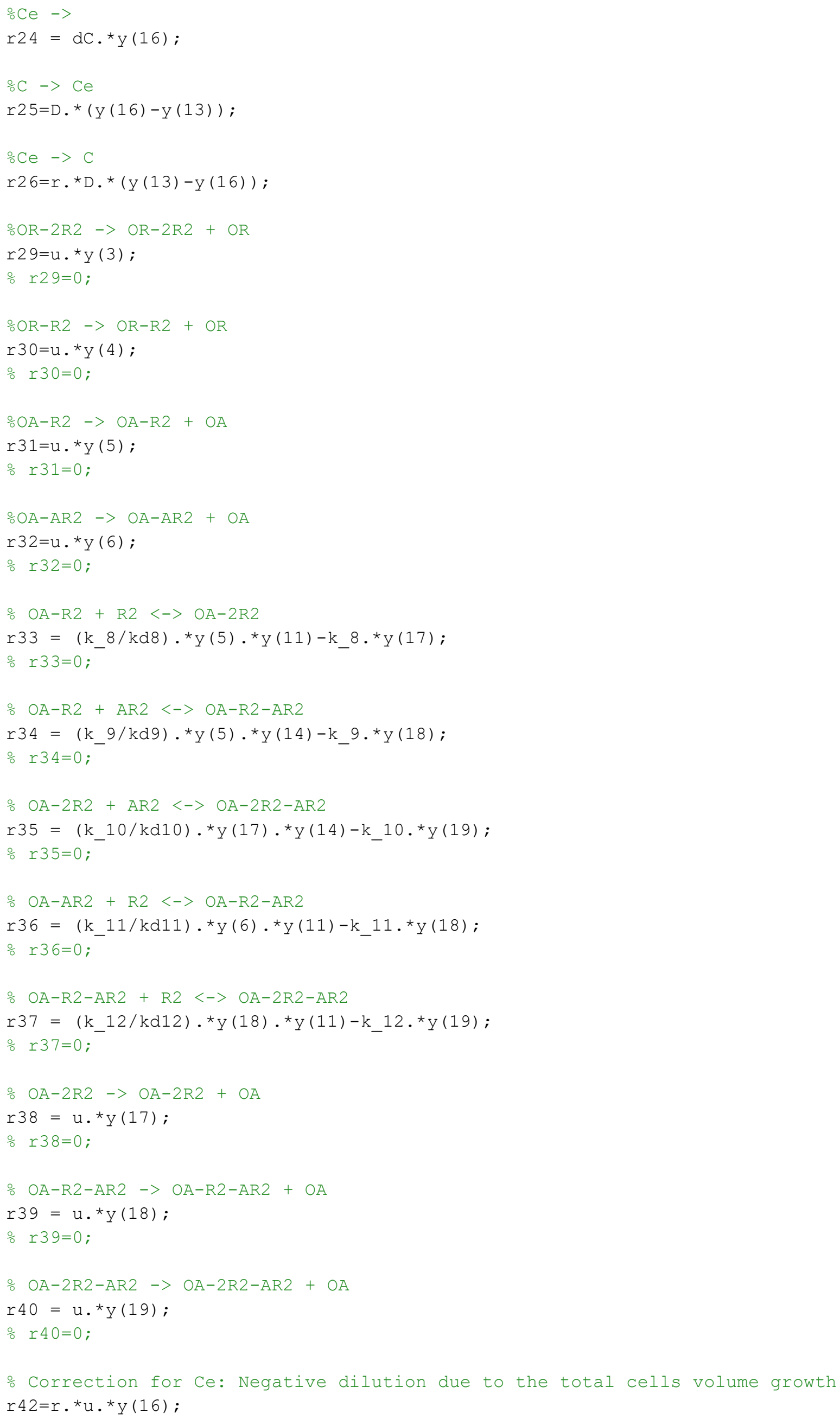


oDifferential equations:

$d y d t=[r 30-r 1+r 29 ; \ldots . \circ \mathrm{OR}$

$r 31+r 32+r 38+r 39+r 40-r 2-r 5 ; \ldots \circ O A$

$r 8-r 29 ; \ldots \% \mathrm{OR}-2 \mathrm{R} 2$

$r 1-r 8-r 30 ; \ldots \% \mathrm{OR}-\mathrm{R} 2$

$r 2-r 31-r 33-r 34 ; \ldots . \circ \mathrm{OA}-\mathrm{R} 2$

$r 5-r 32-r 36 ; \ldots \circ \mathrm{OA}-\mathrm{AR} 2$

$r 7-r 11-r 15 ; \ldots \circ \div$

$r 9+r 10-r 11-r 18 ; \ldots$.

$r 11-r 23 ; \ldots \circ \frac{\circ}{\circ}-a$

$r 12-r 14-r 16 ; \ldots \circ \mathrm{R}$

$r 14-r 1-r 2-r 3-r 4-r 8-r 17-r 33-r 36-r 37 ; \ldots$ ․ R2

$r 13-r 3-r 19 ; \ldots$. $\mathrm{A}$

$r 6-r 4-r 20+r 25 ; \ldots$. $\mathrm{C}$

$r 3-r 5-r 22-r 34-r 35 ; \ldots$ AR2

$r 4-r 21 ; \ldots \circ \mathrm{C} 2-\mathrm{R} 2$

$r 26-r 24+r 42 ; \ldots \quad \div \mathrm{Ce}$

$r 33-r 35-r 38 ; \ldots \circ \mathrm{OA}-2 \mathrm{R} 2$

$r 34+r 36-r 37-r 39 ; \ldots$ O OA-R2-AR2

$r 35+r 37-r 40] ; \% O A-2 R 2-A R 2$

end 


\section{Scenario F}

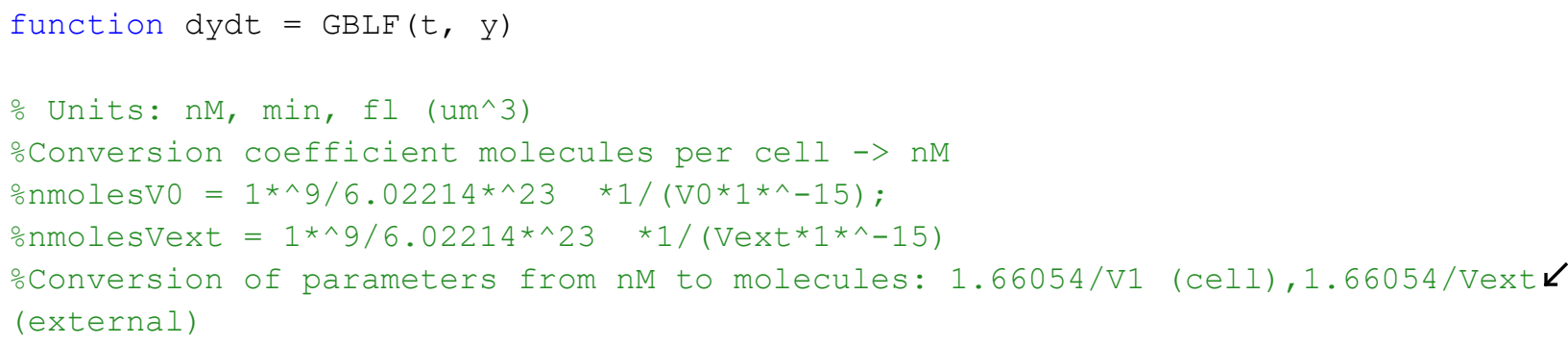

$\% \circ$

$\therefore$ chiset $=1$

$\mathrm{F}=\log (\mathrm{No})+\operatorname{umax} \cdot *(\mathrm{t}-\mathrm{l}+(\log (1-\exp (-\mathrm{v} \cdot \star \mathrm{t})+\exp (-\mathrm{v} \cdot *(\mathrm{t}-\mathrm{l}))) \cdot / \mathrm{v}))-(1 / \mathrm{m}) \cdot{ }^{*} \log (1+((\exp (\mathrm{m} \cdot \boldsymbol{k}$ $\left.\left.\left.\left.\star \operatorname{umax} . *\left(t-1+\left(\log \left(1-\exp \left(-\mathrm{v} \cdot{ }^{*} \mathrm{t}\right)+\exp \left(-\mathrm{v} \cdot{ }^{*}(\mathrm{t}-\mathrm{l})\right)\right) \cdot / \mathrm{v}\right)\right)\right)-1\right) /(\exp (\mathrm{m} \cdot *(\log (\mathrm{K})-\log (\mathrm{No}))))\right)\right)$; $\mathrm{N}=\exp (\mathrm{F}) ;$

$\mathrm{u}=\operatorname{umax} \cdot{ }^{\star}\left(\left(\mathrm{v} \cdot{ }^{\star} \exp \left(-\mathrm{t} \cdot{ }^{\star} \mathrm{v}\right)-\mathrm{v} \cdot{ }^{*} \exp \left(\mathrm{v} \cdot{ }^{*}(1-\mathrm{t})\right)\right) \cdot /\left(\mathrm{v} \cdot{ }^{\star}\left(\exp \left(\mathrm{v} \cdot{ }^{\star}(1-\mathrm{t})\right)-\exp \left(-\mathrm{t} \cdot{ }^{*} \mathrm{v}\right)+1\right)\right)\right.$ $+1)-\left(\operatorname{umax} \cdot{ }^{*} \exp \left(\mathrm{m} \cdot{ }^{*} \operatorname{umax} \cdot{ }^{*}\left(t-1+\log \left(\exp \left(\mathrm{v} \cdot{ }^{*}(\boldsymbol{l}-\mathrm{t})\right)-\exp \left(-\mathrm{t} \cdot{ }^{*} \mathrm{v}\right)+1\right) \cdot / \mathrm{v}\right)\right){ }^{\star} \exp (-\mathrm{m} \cdot \boldsymbol{K}\right.$ $\left.{ }^{*}(\log (\mathrm{K})-\log (\mathrm{NO}))\right) \cdot{ }^{*}\left(\left(\mathrm{v} \cdot{ }^{*} \exp \left(-\mathrm{t} \cdot{ }^{*} \mathrm{~V}\right)-\mathrm{v} \cdot{ }^{*} \exp \left(\mathrm{v} \cdot{ }^{*}(1-\mathrm{t})\right)\right) \cdot /\left(\mathrm{v} \cdot{ }^{*}\left(\exp \left(\mathrm{v}^{*}(1-\mathrm{t})\right)-\exp \boldsymbol{L}\right.\right.\right.$ $\left.\left.\left.\left.\left(-\mathrm{t} \cdot{ }^{*} \mathrm{~V}\right)+1\right)\right)+1\right)\right) \cdot /\left(\exp \left(-\mathrm{m} \cdot{ }^{*}(\log (\mathrm{K})-\log (\mathrm{No}))\right) \cdot{ }^{\star}\left(\exp \left(\mathrm{m} \cdot{ }^{*} \operatorname{umax} \cdot{ }^{*}\left(\mathrm{t}-1+\log \left(\exp \left(\mathrm{v} \cdot{ }^{*} \boldsymbol{L}\right.\right.\right.\right.\right.\right.$ $\left.\left.\left.\left.(1-t))-\exp \left(-t \cdot{ }^{*} \mathrm{v}\right)+1\right) \cdot(\mathrm{v})\right)-1\right)+1\right) ;$

$\mathrm{Vo}=1.2$

$\mathrm{VC}$ tot $=\mathrm{N} * \mathrm{Vo}$

Vtot $=1.8 e+15$;

Vext $=$ Vtot $-V_{C}$ tot;

$\mathrm{Vx}=\mathrm{Vext} . / \mathrm{VC}_{\mathrm{C}}$ tot;

$r=1 . / V x$;

$\mathrm{kFR}=\mathrm{kF}{ }^{*} \mathrm{chi}$;

ㅇ $\mathrm{kFR}=\mathrm{kF}^{*}$ chiset;

$\mathrm{kFA}=\mathrm{kF} / \mathrm{chi}$; 


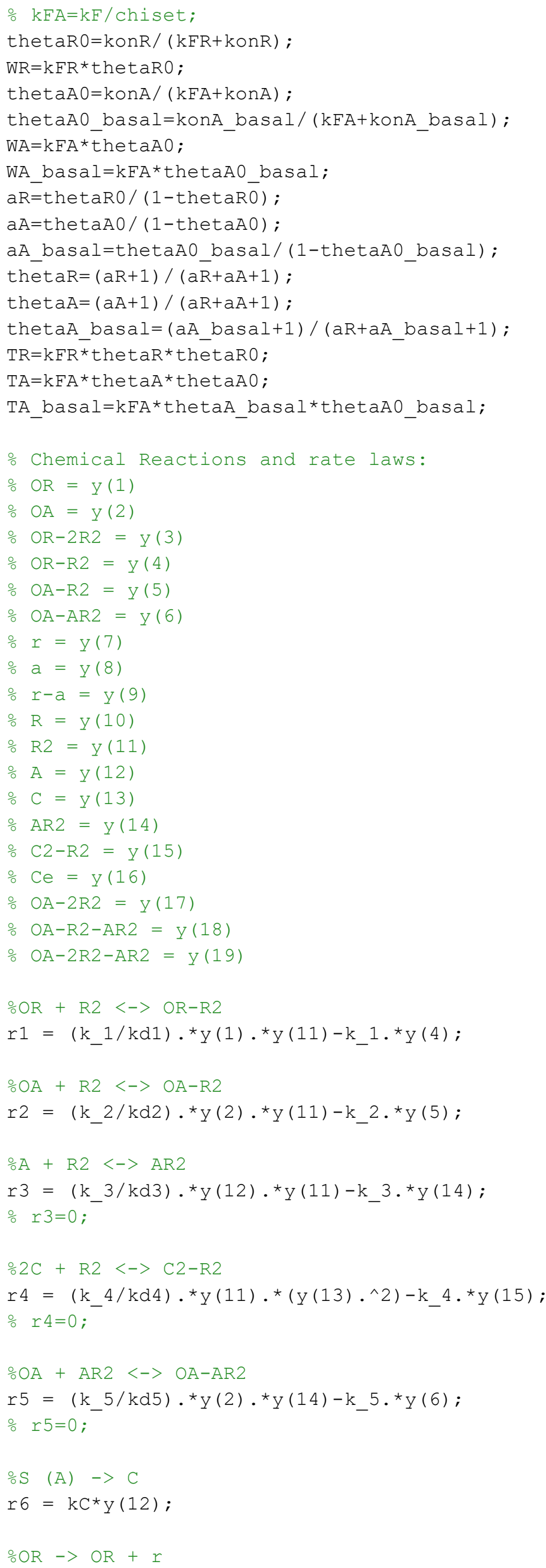




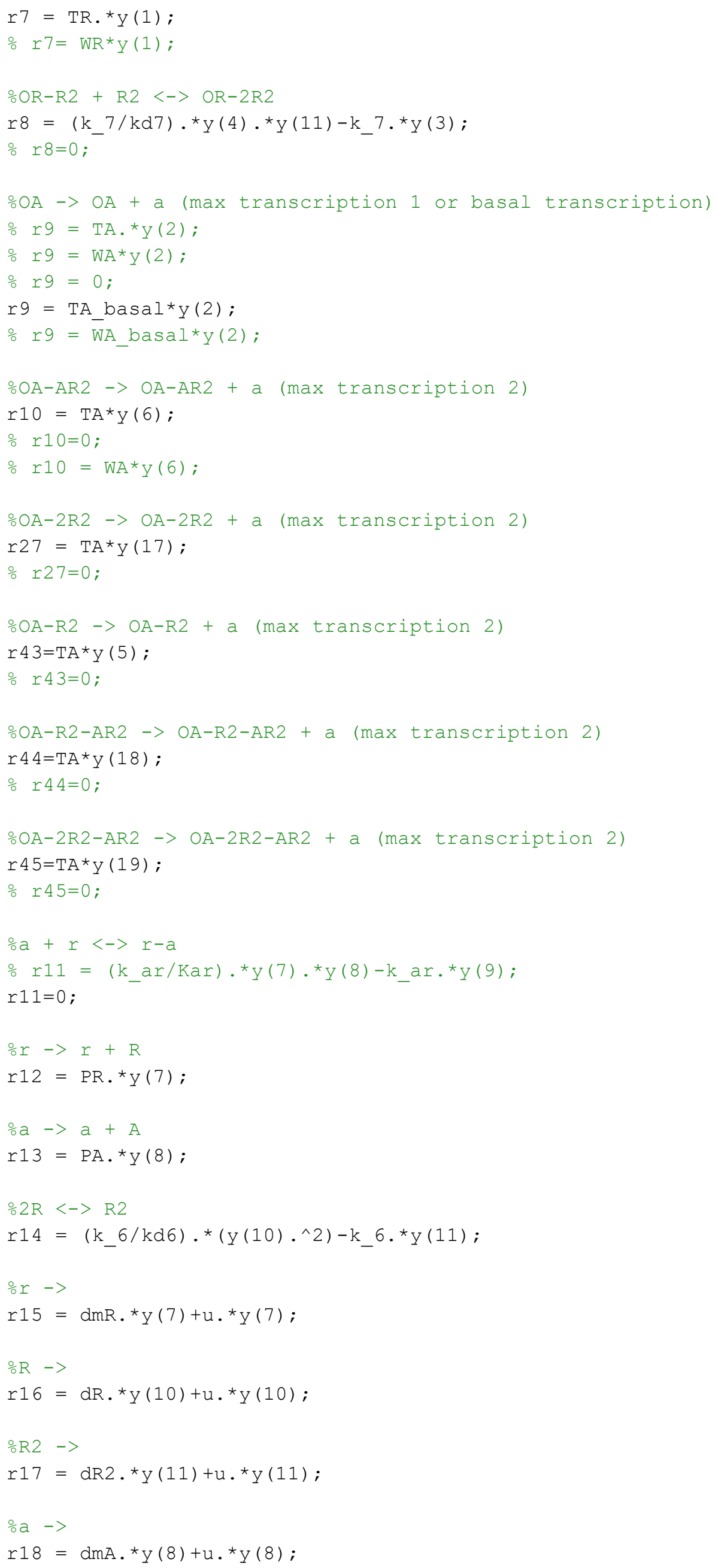




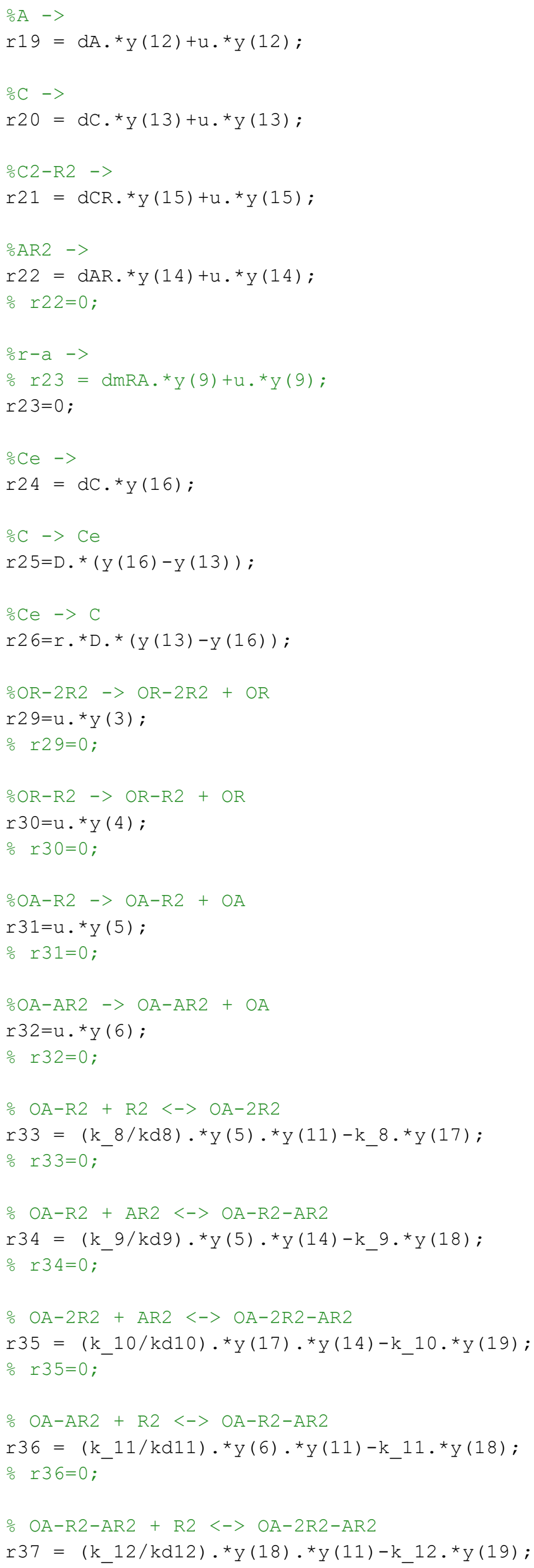




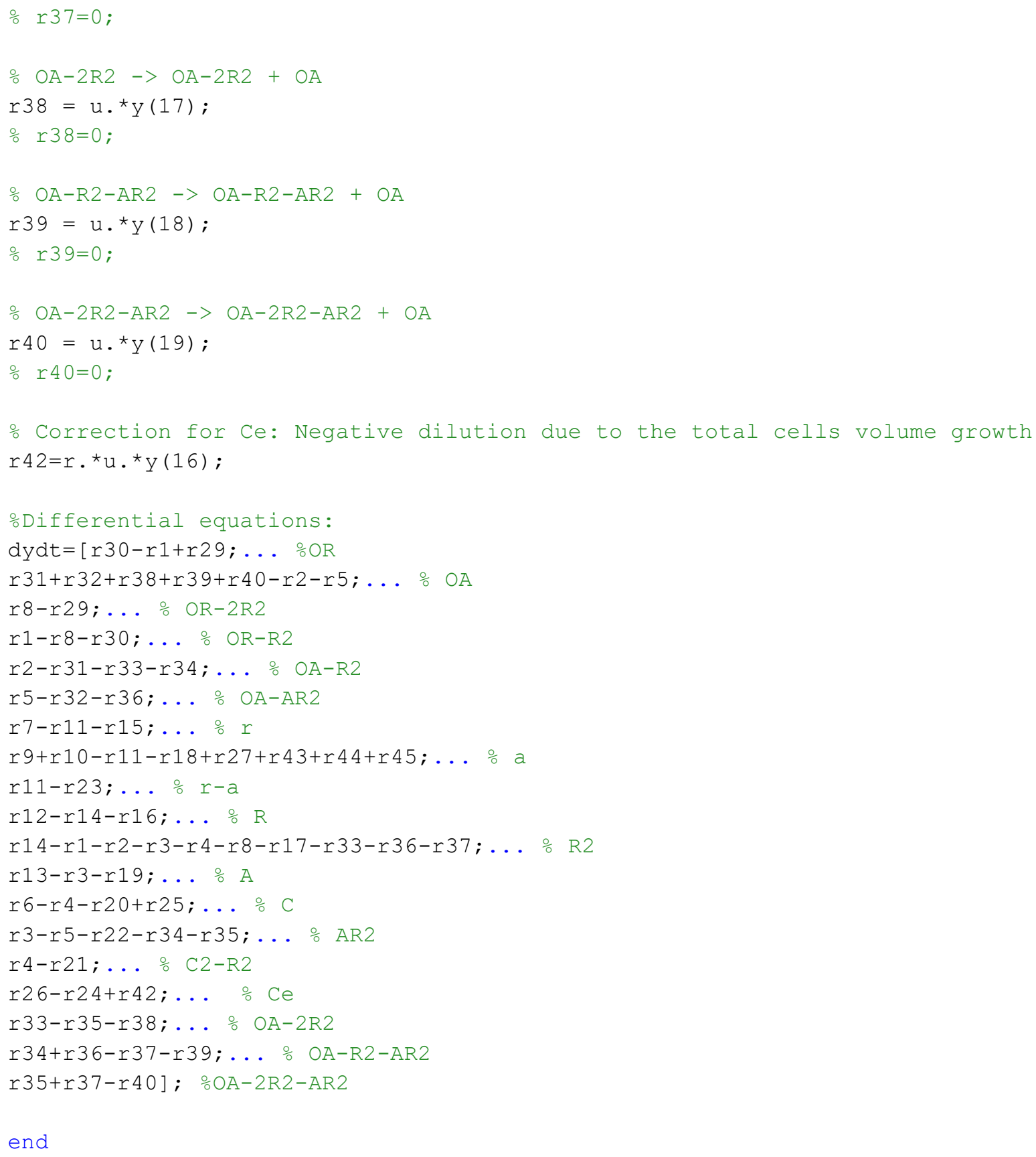

end 


\section{Scenario G}

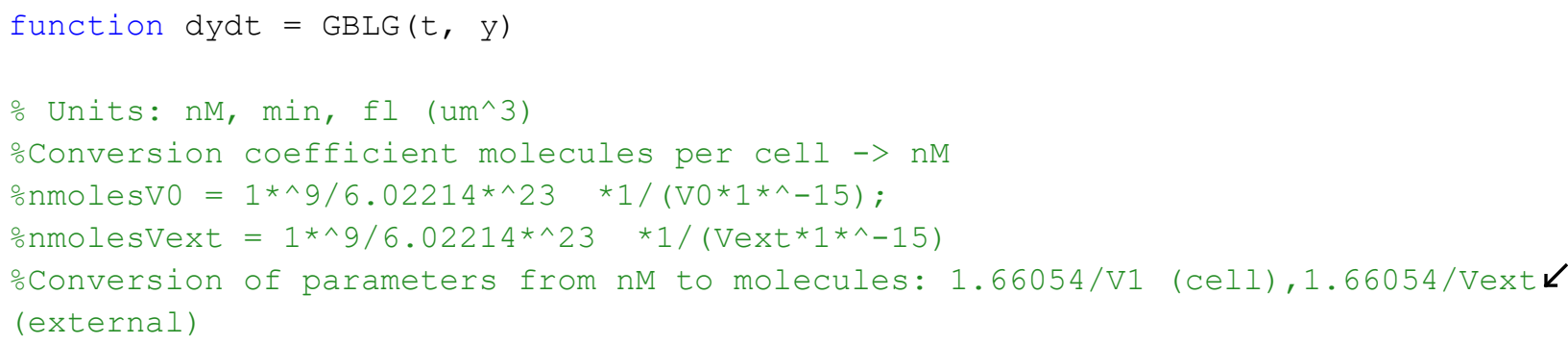




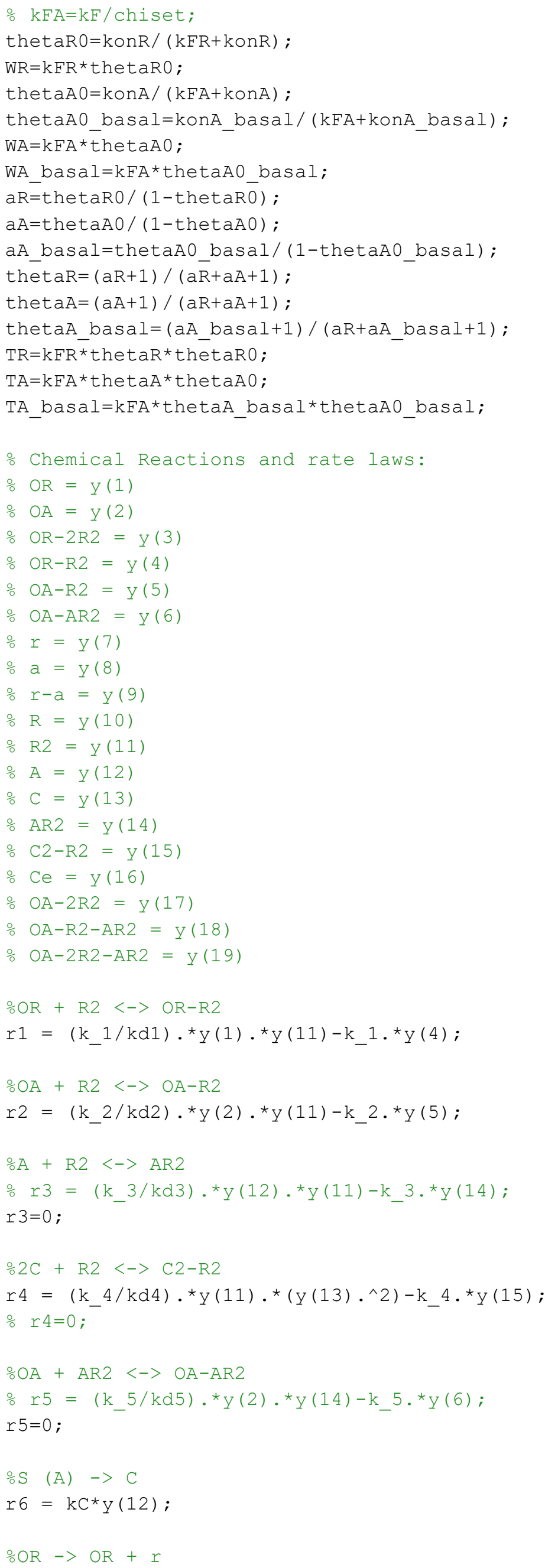




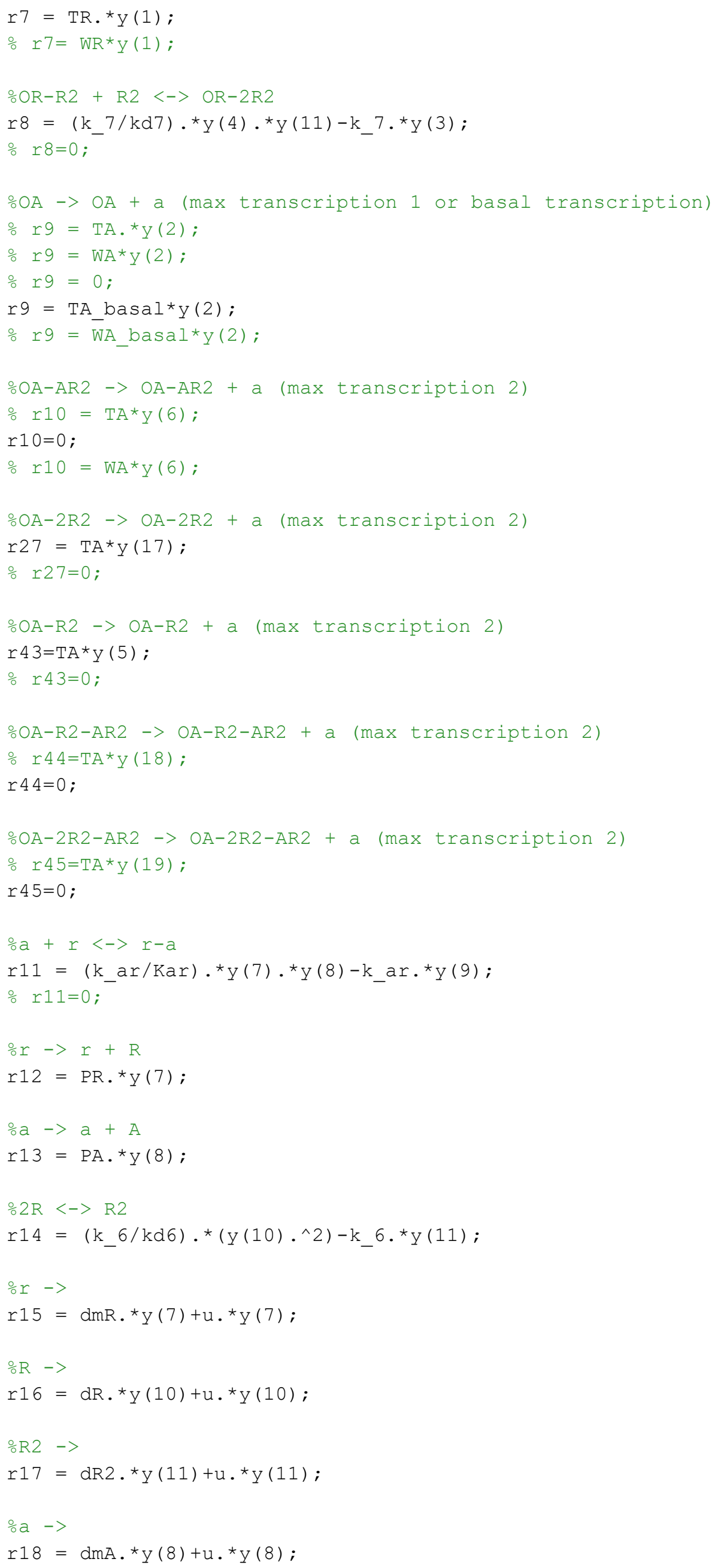




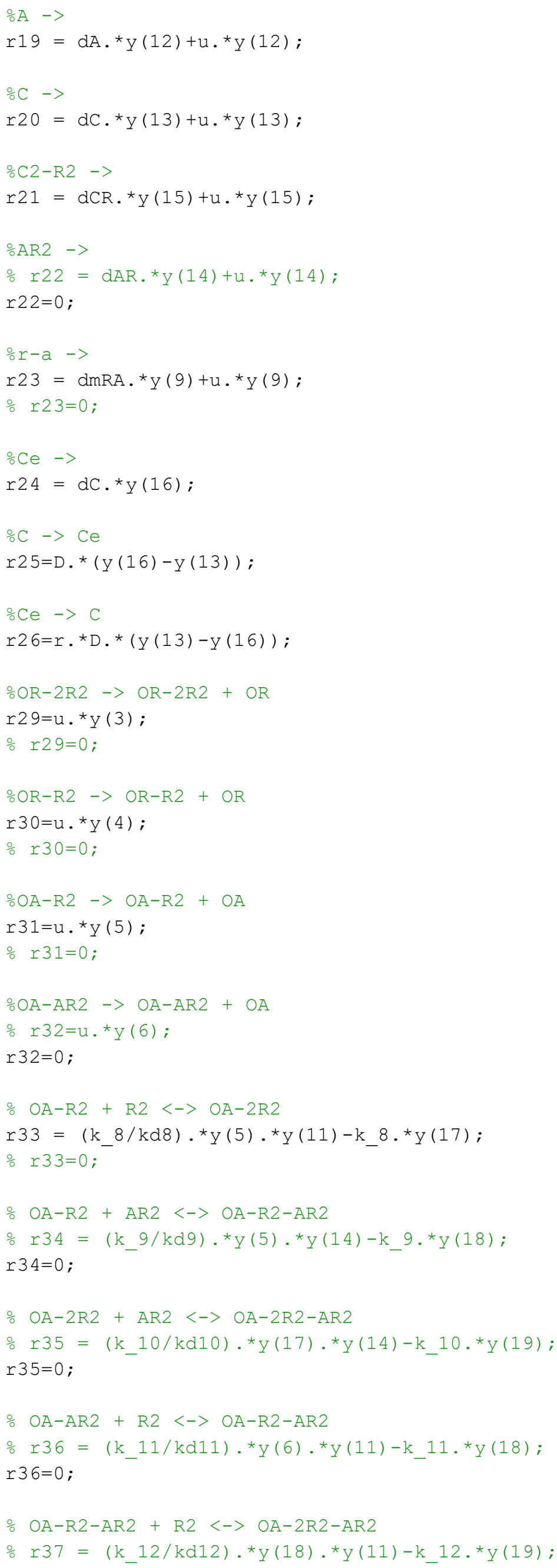


$r 37=0$

$\div \mathrm{OA}-2 \mathrm{R} 2->\mathrm{OA}-2 \mathrm{R} 2+\mathrm{OA}$

$\mathrm{r38}=\mathrm{u} \cdot{ }^{\star} \mathrm{y}(17)$;

당 0 ;

$\div \mathrm{OA}-\mathrm{R} 2-\mathrm{AR} 2->\mathrm{OA}-\mathrm{R} 2-\mathrm{AR} 2+\mathrm{OA}$

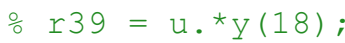

$r 39=0$

$\circ \mathrm{OA}-2 \mathrm{R} 2-\mathrm{AR} 2->\mathrm{OA}-2 \mathrm{R} 2-\mathrm{AR} 2+\mathrm{OA}$

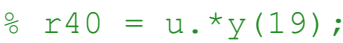

$r 40=0$;

o Correction for Ce: Negative dilution due to the total cells volume growth $r 42=r \cdot{ }^{*} u \cdot{ }^{\star} \mathrm{y}(16) ;$

Differential equations:

$d y d t=[r 30-r 1+r 29 ; \ldots . \circ \mathrm{OR}$

$r 31+r 32+r 38+r 39+r 40-r 2-r 5 ; \ldots \% O A$

$r 8-r 29 ; \ldots \circ \mathrm{OR}-2 \mathrm{R} 2$

$r 1-r 8-r 30 ; \ldots \circ \mathrm{OR}-\mathrm{R} 2$

$r 2-r 31-r 33-r 34 ; \ldots . \div \mathrm{OA}-\mathrm{R} 2$

$r 5-r 32-r 36 ; \ldots \circ \mathrm{OA}-\mathrm{AR} 2$

$r 7-r 11-r 15 ; \ldots \circ \mathrm{\circ}$

$r 9+r 10-r 11-r 18+r 27+r 43+r 44+r 45 ; \ldots$ a

$r 11-r 23 ; \ldots . . \div-a$

$r 12-r 14-r 16 ; \ldots \% \mathrm{R}$

$r 14-r 1-r 2-r 3-r 4-r 8-r 17-r 33-r 36-r 37 ; \ldots$ 응 2

$r 13-r 3-r 19 ; \ldots \circ \mathrm{A}$

$r 6-r 4-r 20+r 25 ; \ldots \circ C$

$r 3-r 5-r 22-r 34-r 35 ; \ldots \circ$ AR2

$r 4-r 21 ; \ldots \circ \mathrm{C} 2-\mathrm{R} 2$

$r 26-r 24+r 42 ; \ldots \quad \% \mathrm{Ce}$

$r 33-r 35-r 38 ; \ldots \circ \mathrm{OA}-2 \mathrm{R} 2$

$r 34+r 36-r 37-r 39 ; \ldots \circ \mathrm{OA}-\mathrm{R} 2-\mathrm{AR} 2$

$r 35+r 37-r 40] ; \div O A-2 R 2-A R 2$

end 


\section{Scenario $\mathrm{H}$}

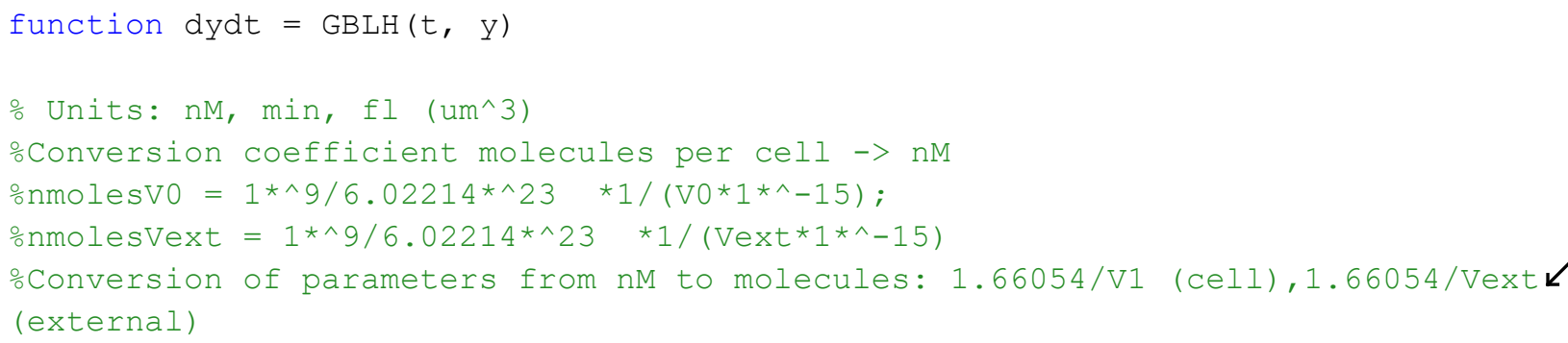

응 $\frac{\circ}{0}$

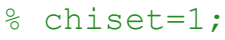

$\mathrm{F}=\log (\mathrm{No})+\operatorname{umax} \cdot *(\mathrm{t}-\mathrm{l}+(\log (1-\exp (-\mathrm{v} \cdot * \mathrm{t})+\exp (-\mathrm{v} \cdot *(\mathrm{t}-\mathrm{l}))) \cdot / \mathrm{v}))-(1 / \mathrm{m}) \cdot{ }^{*} \log (1+((\exp (\mathrm{m} \cdot \boldsymbol{k}$ $\left.\left.\left.\left.{ }^{\star} \operatorname{umax} .{ }^{*}\left(t-l+\left(\log \left(1-\exp \left(-\mathrm{v} \cdot{ }^{*} \mathrm{t}\right)+\exp \left(-\mathrm{v} \cdot{ }^{\star}(\mathrm{t}-\mathrm{l})\right)\right) \cdot / \mathrm{v}\right)\right)\right)-1\right) /\left(\exp \left(\mathrm{m} \cdot{ }^{*}(\log (\mathrm{K})-\log (\mathrm{No}))\right)\right)\right)\right)$; $\mathrm{N}=\exp (\mathrm{F}) ;$

$\mathrm{u}=\operatorname{umax} \cdot{ }^{*}\left(\left(\mathrm{v} \cdot{ }^{\star} \exp \left(-\mathrm{t} \cdot{ }^{\star} \mathrm{v}\right)-\mathrm{v} \cdot{ }^{*} \exp \left(\mathrm{v} \cdot{ }^{*}(1-\mathrm{t})\right)\right) \cdot /\left(\mathrm{v} \cdot{ }^{\star}\left(\exp \left(\mathrm{v} \cdot{ }^{*}(1-\mathrm{t})\right)-\exp \left(-\mathrm{t} \cdot{ }^{*} \mathrm{v}\right)+1\right)\right)\right.$ $+1)-\left(\operatorname{umax} \cdot{ }^{*} \exp \left(\mathrm{m} \cdot{ }^{*} \operatorname{umax} \cdot{ }^{*}\left(\mathrm{t}-\mathbf{l}+\log \left(\exp \left(\mathrm{v} \cdot{ }^{*}(1-\mathrm{t})\right)-\exp \left(-\mathrm{t} \cdot{ }^{*} \mathrm{v}\right)+1\right) \cdot / \mathrm{v}\right)\right){ }^{\star} \exp (-\mathrm{m} \cdot \boldsymbol{K}\right.$ $\star(\log (\mathrm{K})-\log (\mathrm{NO}))) \cdot{ }^{\star}\left(\left(\mathrm{v} \cdot{ }^{\star} \exp \left(-\mathrm{t} \cdot{ }^{\star} \mathrm{v}\right)-\mathrm{v} \cdot{ }^{\star} \exp \left(\mathrm{v} \cdot{ }^{\star}(1-\mathrm{t})\right)\right) \cdot /\left(\mathrm{v} \cdot{ }^{\star}\left(\exp \left(\mathrm{v}^{\star}(1-\mathrm{t})\right)-\exp \swarrow\right.\right.\right.$ $\left.\left.\left.\left.\left(-\mathrm{t} \cdot{ }^{\star} \mathrm{V}\right)+1\right)\right)+1\right)\right) \cdot /\left(\exp \left(-\mathrm{m} \cdot{ }^{*}(\log (\mathrm{K})-\log (\mathrm{No}))\right) \cdot{ }^{*}\left(\exp \left(\mathrm{m} \cdot{ }^{*} \operatorname{umax} \cdot{ }^{*}\left(\mathrm{t}-1+\log \left(\exp \left(\mathrm{v} \cdot{ }^{\star} \boldsymbol{L}\right.\right.\right.\right.\right.\right.$ $\left.\left.\left.\left.(1-t))-\exp \left(-t \cdot{ }^{*} \mathrm{v}\right)+1\right) \cdot(\mathrm{v})\right)-1\right)+1\right) ;$

$\mathrm{Vo}=1.2$

$\mathrm{VC}$ tot $=\mathrm{N} * \mathrm{Vo}$

Vtot $=1.8 e+15$;

Vext $=V$ tot $-V_{C}$ tot;

$\mathrm{Vx}=\mathrm{Vext} . / \mathrm{VC}_{\mathrm{C}}$ tot;

$\mathrm{r}=1 . / \mathrm{Vx}$;

$\mathrm{kFR}=\mathrm{kF}{ }^{*} \mathrm{chi}$;

$\circ \mathrm{kFR}=\mathrm{kF}^{*} \mathrm{chiset}$;

$\mathrm{kFA}=\mathrm{kF} / \mathrm{chi}$; 


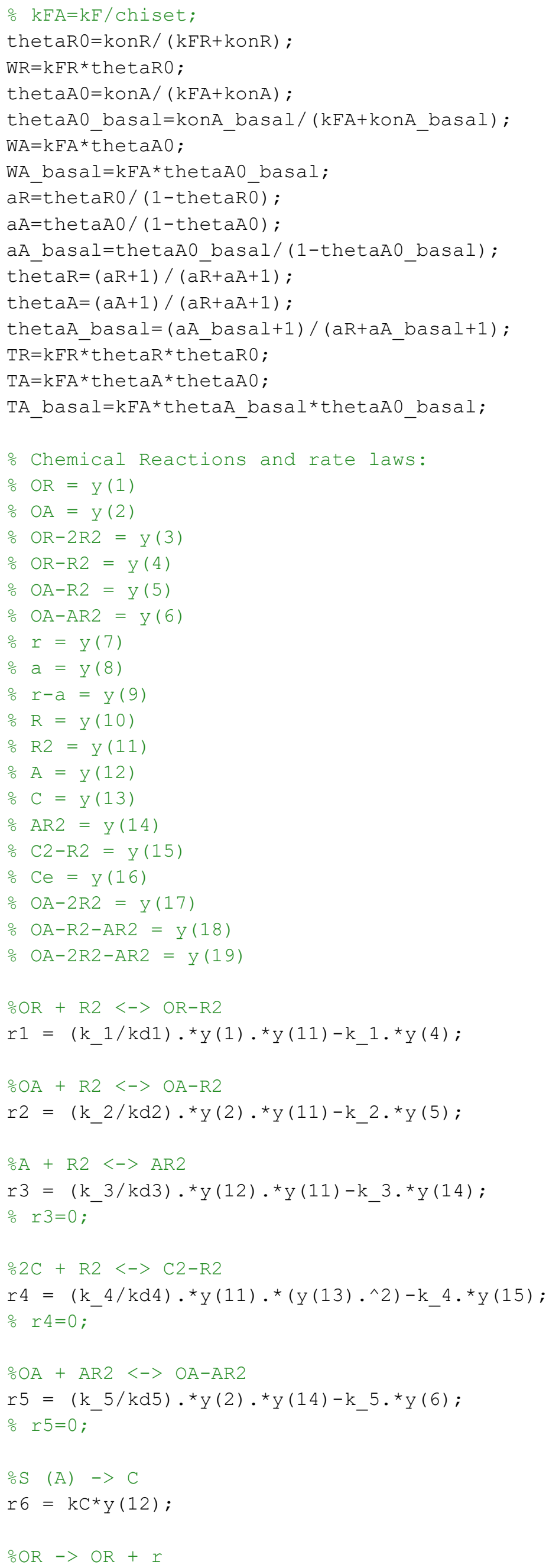




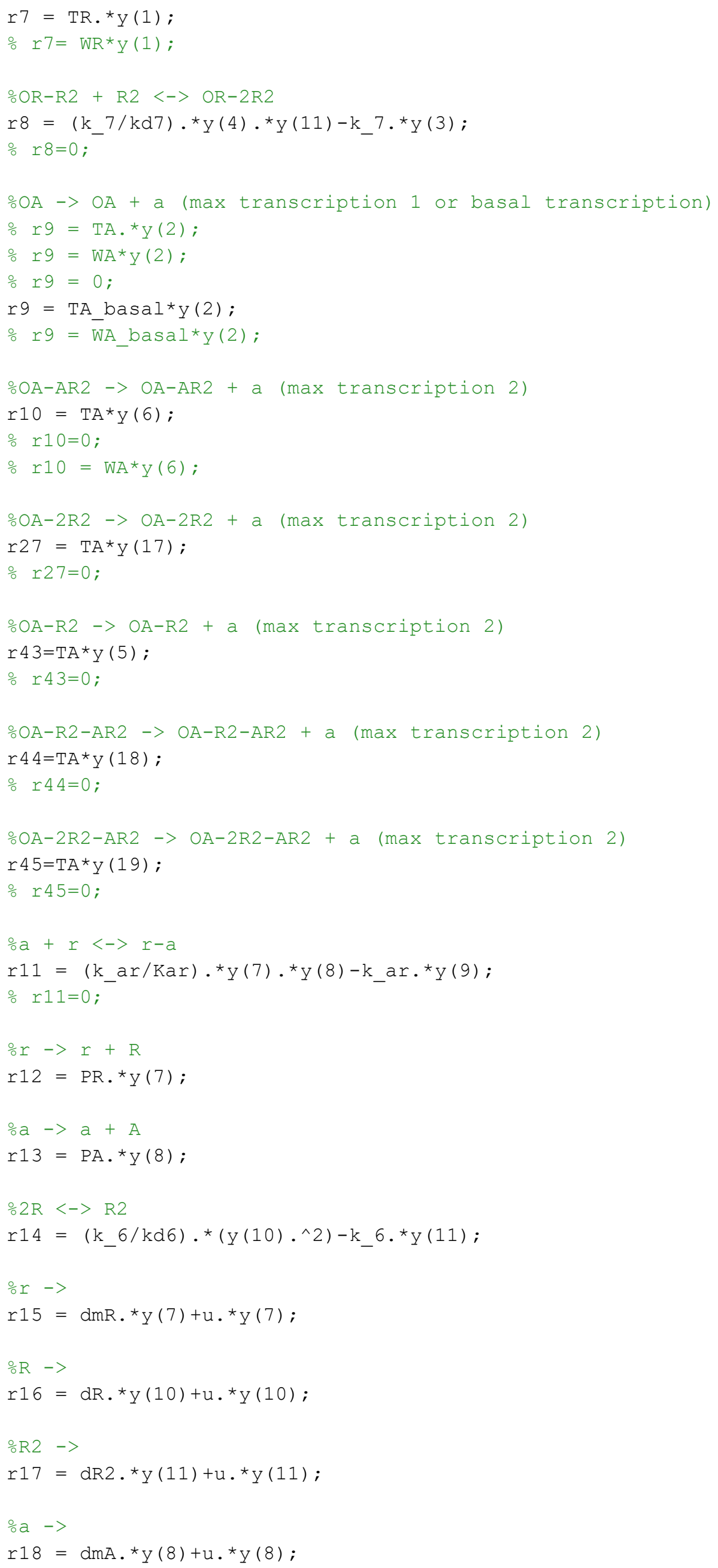




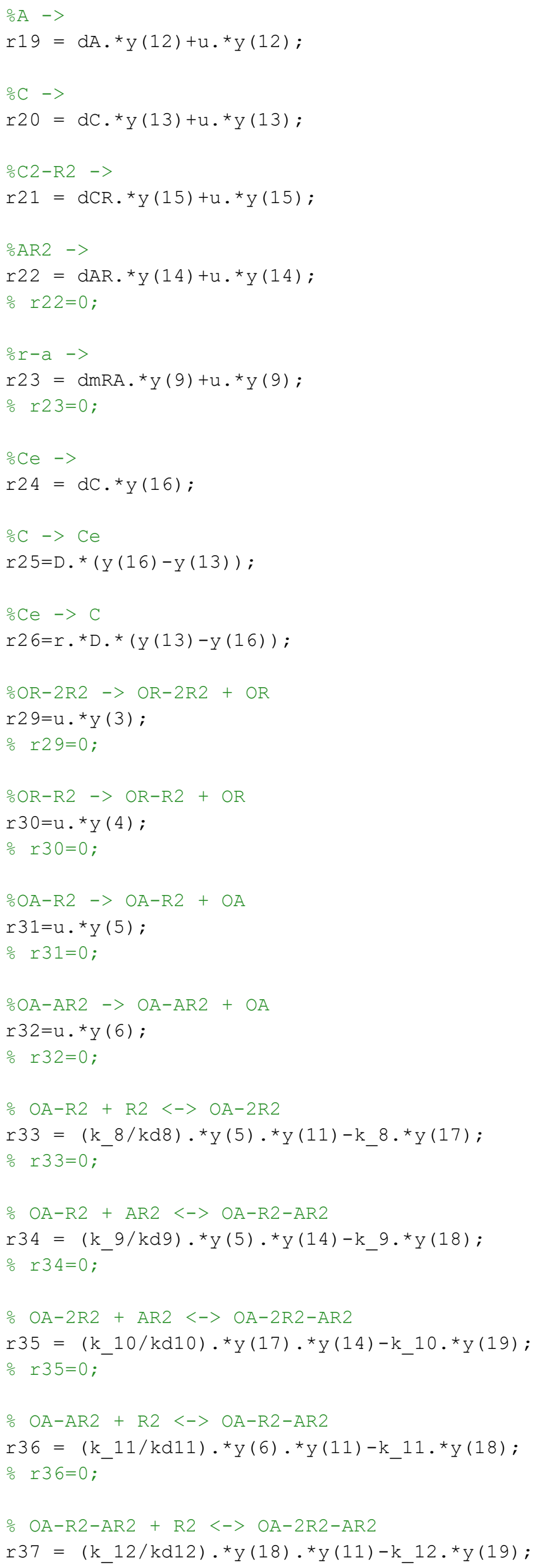




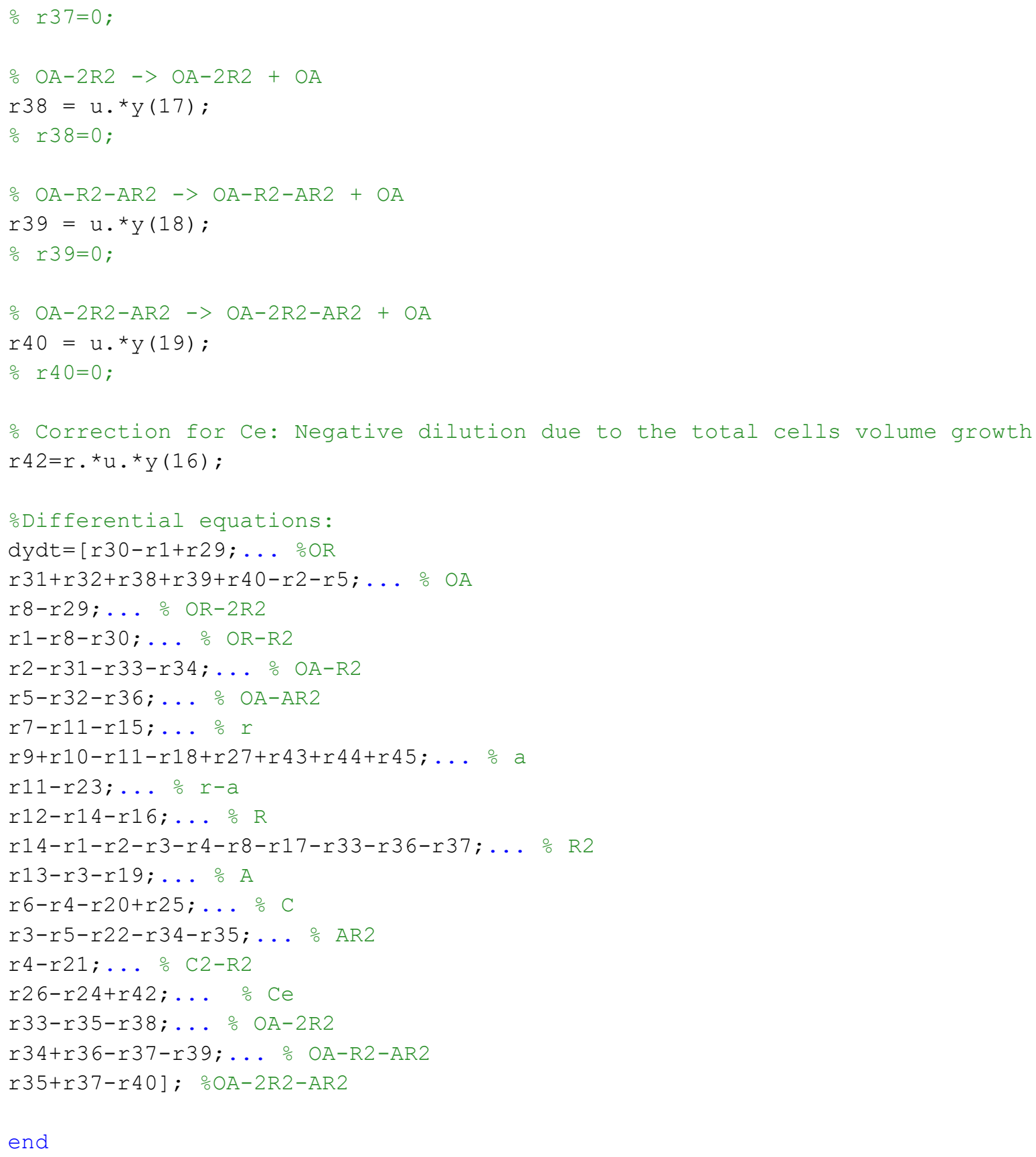

end 